Ann. Scient. Éc. Norm. Sup.,

$4^{\text {e }}$ série, t. 39, 2006, p. 467 à 511.

\title{
DIMENSIONS OF SOME AFFINE DELIGNE-LUSZTIG VARIETIES
}

\author{
By UlRICH GÖRTZ, ThOMAS J. HAINES ${ }^{1}$, ROBERT E. KOTTWITZ ${ }^{2}$ \\ AND DANIEL C. REUMAN ${ }^{3}$
}

\begin{abstract}
This paper concerns the dimensions of certain affine Deligne-Lusztig varieties, both in the affine Grassmannian and in the affine flag manifold. Rapoport conjectured a formula for the dimensions of the varieties $X_{\mu}(b)$ in the affine Grassmannian. We prove his conjecture for $b$ in the split torus; we find that these varieties are equidimensional; and we reduce the general conjecture to the case of superbasic $b$. In the affine flag manifold, we prove a formula that reduces the dimension question for $X_{x}(b)$ with $b$ in the split torus to computations of dimensions of intersections of Iwahori orbits with orbits of the unipotent radical. Calculations using this formula allow us to verify a conjecture of Reuman in many new cases, and to make progress toward a generalization of his conjecture.
\end{abstract}

(c) 2006 Elsevier Masson SAS

RÉSUMÉ. - Cet article concerne les dimensions de certaines variétés de Deligne-Lusztig affines, dans la grassmannienne affine et dans la variété de drapeaux affine. Rapoport a conjecturé une formule pour les dimensions des variétés $X_{\mu}(b)$ dans la grassmannienne affine. On prouve cette conjecture pour $b$ dans le tore déployé ; on trouve que ces variétés sont équidimensionnelles; et on réduit la conjecture générale au cas où $b$ est «super-basique». Dans le cas de la variété de drapeaux affine, on prouve une formule qui réduit la détermination de la dimension de $X_{x}(b)$ avec $b$ dans le tore déployé au calcul de dimensions d'intersections d'orbites du sous-groupe d'Iwahori et d'orbites du radical unipotent. En utilisant cette formule on vérifie une conjecture de Reuman dans beaucoup de cas nouveaux, et on généralise sa conjecture.

(c) 2006 Elsevier Masson SAS

\section{Introduction}

Let $k$ be a finite field with $q$ elements, and let $\bar{k}$ be an algebraic closure of $k$. We consider the field $L:=\bar{k}((\varepsilon))$ and its subfield $F:=k((\varepsilon))$. We write $\sigma: x \mapsto x^{q}$ for the Frobenius automorphism of $\bar{k} / k$, and we also regard $\sigma$ as an automorphism of $L / F$ in the usual way, so that $\sigma\left(\sum a_{n} \varepsilon^{n}\right)=\sum \sigma\left(a_{n}\right) \varepsilon^{n}$. We write $\mathfrak{o}$ for the valuation ring $\bar{k}[[\varepsilon]]$ of $L$.

Let $G$ be a split connected reductive group over $k$, and let $A$ be a split maximal torus of $G$. Put $\mathfrak{a}:=X_{*}(A)_{\mathbb{R}}$. Write $W$ for the Weyl group of $A$ in $G$. Fix a Borel subgroup $B=A U$ containing $A$ with unipotent radical $U$. For $\lambda \in X_{*}(A)$ we write $\varepsilon^{\lambda}$ for the element of $A(F)$ obtained as the image of $\varepsilon \in \mathbb{G}_{m}(F)$ under the homomorphism $\lambda: \mathbb{G}_{m} \rightarrow A$.

This paper concerns the dimensions of certain affine Deligne-Lusztig varieties, both in the affine Grassmannian and in the affine flag manifold. We begin with the affine Grassmannian.

\footnotetext{
${ }^{1}$ Haines was partially supported by NSF Grant DMS-0303605 and a Sloan Research Fellowship.

${ }^{2}$ Kottwitz was partially supported by NSF Grant DMS-0245639.

${ }^{3}$ Reuman was supported by NSF Grants DEB-9981552 and DMS-0443803.
} 
Put $K:=G(\mathfrak{o})$. We denote by $X$ the affine Grassmannian $X=G(L) / K$ and by $x_{0}$ its obvious base-point. The group $G(L)$ acts by left translation on $X$. By the Cartan decomposition $G(L)$ is the disjoint union of the subsets $K \varepsilon^{\mu} K$, with $\mu$ running over the dominant elements in $X_{*}(A)$. For $b \in G(L)$ and a dominant coweight $\mu \in X_{*}(A)$ the affine Deligne-Lusztig variety $X_{\mu}(b)=X_{\mu}^{G}(b)$ is the locally closed subset of $X$ defined by

$$
X_{\mu}(b):=\left\{x \in G(L) / K: x^{-1} b \sigma(x) \in K \varepsilon^{\mu} K\right\}
$$

For $g \in G(L)$ it is clear that $x \mapsto g x$ yields an isomorphism $X_{\mu}(b) \rightarrow X_{\mu}\left(g b \sigma(g)^{-1}\right)$, so the isomorphism class of $X_{\mu}(b)$ depends only on the $\sigma$-conjugacy class of $b$.

Let $\mathbb{D}$ be the diagonalizable group over $F$ with character group $\mathbb{Q}$. Just as in [13], the element $b$ determines a homomorphism $\nu_{b}: \mathbb{D} \rightarrow G$ over $L$, and $b$ is said to be basic if $\nu_{b}$ factors through the center of $G$. For $g \in G(L)$ we have

$$
\nu_{g b \sigma(g)^{-1}}=\operatorname{Int}(g) \circ \nu_{b}
$$

where Int $(g)$ denotes the inner automorphism $x \mapsto g x g^{-1}$ of $G$ over $L$, and since it is harmless to $\sigma$-conjugate $b$, we may as well assume that $\nu_{b}$ factors through $A$, and that the corresponding element $\bar{\nu}_{b} \in X_{*}(A)_{\mathbb{Q}} \subset \mathfrak{a}$ is dominant. Following [29] we refer to $\bar{\nu}_{b}$ as the Newton point of $b$. The centralizer $M_{b}$ of $\nu_{b}$ in $G$ is then a Levi subgroup of $G$ over $F$.

Just as in [13], [30, 1.12], [14], there is an inner form $J$ of $M_{b}$ whose $R$-valued points (for any $F$-algebra $R$ ) are given by

$$
J(R)=\left\{g \in G\left(R \otimes_{F} L\right): g^{-1} b \sigma(g)=b\right\} .
$$

The group $J(F)$ acts by left multiplication on $X_{\mu}(b)$. Note that $J(F) \subset M_{b}(L)$ because of (1.0.2).

Let $\Lambda_{G}$ denote the quotient of $X_{*}(A)$ by the coroot lattice for $G$. We denote by $p_{G}$ the canonical surjection $X_{*}(A) \rightarrow \Lambda_{G}$. There is a canonical homomorphism $\eta_{G}: G(L) \rightarrow \Lambda_{G}$, which is trivial on $K=G(\mathfrak{o})$ and hence induces a surjection, also denoted $\eta_{G}$, from $X=$ $G(L) / K$ to $\Lambda_{G}$. The fibers of $\eta_{G}: X \rightarrow \Lambda_{G}$ are the connected components of the affine Grassmannian $X$. As in [14, 7.7], the restriction of the homomorphism $\eta_{M_{b}}: M_{b}(L) \rightarrow \Lambda_{M_{b}}$ to $J(F)$ is surjective, which implies that the restriction of $\eta_{G}: G(L) \rightarrow \Lambda_{G}$ to $J(F)$ is also surjective. Using the action of $J(F)$ on $X_{\mu}(b)$, we then see that the intersections $X_{\mu}(b) \cap \eta_{G}^{-1}(\lambda)$ $\left(\lambda \in \Lambda_{G}\right)$ of $X_{\mu}(b)$ with the various connected components of $X$ are all isomorphic to each other.

As in [16] (see also [17,15]), there is a simple criterion for $X_{\mu}(b)$ to be non-empty (see Proposition 5.6.1). Here we mention only that when $b$ is basic, $X_{\mu}(b)$ is non-empty if and only if $\eta_{G}(b)=p_{G}(\mu)$. When $X_{\mu}(b)$ is non-empty, there is a formula for its dimension, conjectured by Rapoport [28], which we now recall, reformulating it slightly (see [12]) along the way.

This formula involves a non-negative integer $\operatorname{def}_{G}(b)$ attached to $b$. By definition $\operatorname{def}_{G}(b)$ is the $F$-rank of $G$ minus the $F$-rank of $J$. Clearly $\operatorname{def}_{G}(b)$ depends only on the $\sigma$-conjugacy class of $b$. (As usual the $F$-rank of $G$ is the common dimension of all maximal $F$-split tori in $G$.) We write $\rho \in X^{*}(A)_{\mathbb{Q}}$ for the half-sum of the positive roots.

Conjecture 1.0.1 (Rapoport). - Assume that $X_{\mu}(b)$ is non-empty. Then its dimension is given by

$4^{\mathrm{e}}$ SÉRIE - TOME $39-2006-\mathrm{N}^{\circ} 3$ 


$$
\operatorname{dim} X_{\mu}(b)=\left\langle\rho, \mu-\bar{\nu}_{b}\right\rangle-\frac{1}{2} \operatorname{def}_{G}(b) .
$$

In [32] Reuman proves the conjecture for $G=S L_{2}, S L_{3}, S p_{4}$ and $b=1$. In [33] Viehmann proves (in the context of $\mathbb{Q}_{p}$ rather than $k((\varepsilon))$ ) that Rapoport's conjecture is true for $G L_{n}$ and minuscule $\mu$.

In this paper we prove Rapoport's conjecture for all $b \in A(L)$ (see Theorem 2.15.1, noting that $\operatorname{def}_{G}(b)=0$ when $b \in A(L)$ ), and in fact we show in this case that $X_{\mu}(b)$ is equidimensional (see Proposition 2.17.1), answering a question of Rapoport. Moreover, returning to general elements $b \in G(L)$, we show in Theorem 5.8.1 that, if $M$ is a Levi subgroup of $G$ such that $b \in M(L)$ and $b$ is basic in $M(L)$, and if Rapoport's conjecture holds for $(M, b)$, then Rapoport's conjecture holds for $(G, b)$.

Consequently, in order to prove Rapoport's conjecture in general, it is enough to prove it for superbasic elements $b$, by which we mean those for which no $\sigma$-conjugate is contained in a proper Levi subgroup of $G$. As we verify in Section 5.9, superbasic elements are very special, and for simple groups they exist only in type $A_{n}$. The upshot is that it is enough to prove Rapoport's conjecture for basic elements $b \in G L_{n}(L)$ such that the valuation of $\operatorname{det}(b)$ is relatively prime to $n$. This has been done, shortly after this paper appeared as a preprint, by Viehmann [34], so Rapoport's conjecture is now a theorem.

Now we turn to affine Deligne-Lusztig varieties inside the affine flag manifold $G(L) / I$, where $I$ is the Iwahori subgroup of $G(L)$ obtained from an alcove $\mathbf{a}_{1}$ in the apartment associated to $A$. Given $b \in G(L)$ and an element $x \in \widetilde{W}=W \ltimes X_{*}(A)$, we get the affine Deligne-Lusztig variety

$$
X_{x}(b)=\left\{g \in G(L) / I: g^{-1} b \sigma(g) \in I x I\right\} .
$$

For the groups $G=S L_{2}, S L_{3}, S p_{4}$ and $b=1$ the dimension of $X_{x}(b)$ was computed by Reuman [32]. For suitably general $x$ (those in the union of the "shrunken Weyl chambers") Reuman gives a simple formula for $\operatorname{dim} X_{x}(b)$ and conjectures that it holds in general (for $b=1$ and suitably general $x$ ).

In this article we prove a formula (see Theorem 6.3.1) for $\operatorname{dim} X_{x}(b)$ when $b \in A(L)$. Unfortunately this formula does not suffice to establish Reuman's conjecture, since it involves the unknown dimension of the intersection of $I$ - and $U(L)$-orbits in the affine flag manifold. (The reason we had better luck with the affine Grassmannian is that the dimensions of intersections of $G(\mathfrak{o})$ - and $U(L)$-orbits in the affine Grassmannian are known, thanks to Mirković and Vilonen [23].)

However there is an algorithm for computing the dimensions of such intersections, and in Section 7 we describe the results of computer calculations made using this algorithm. Reuman's conjecture (see Section 7.2) turns out to hold in all cases checked by the computer. In the case of rank 2 groups, the results can be presented in the form of pictures. Fig. 1 shows the dimensions for $b=1$ and type $G_{2}$, while Figs. 2, 3 show dimensions for two elements $b \in A(L), b \neq 1$, one for type $A_{2}$, and one for type $C_{2}$.

The results in these last two figures support Conjecture 7.5.1, an extension of Reuman's conjecture to elements $b \in A(L)$. We finish this introduction by mentioning that there is mounting evidence that an analog of Reuman's conjecture holds for all $b \in G(L)$ (for suitably general $x \in \widetilde{W}$ ). 


\section{Affine Deligne-Lusztig varieties inside the affine Grassmannian}

\subsection{Further preliminaries}

For $\nu$ in $X_{*}(A)$ or $\mathfrak{a}$ we write $\nu_{\text {dom }}$ for the unique dominant element in the $W$-orbit of $\nu$. For cocharacters $\mu, \nu \in X_{*}(A)$ we say that $\nu \leqslant \mu$ if $\mu-\nu$ is a non-negative integral linear combination of positive coroots.

Any $b \in A(L)$ is $\sigma$-conjugate to an element of the form $\varepsilon^{\nu}$. By [16] the set $X_{\mu}\left(\varepsilon^{\nu}\right)$ is nonempty if and only if $\nu_{\text {dom }} \leqslant \mu$; we now assume that this is the case. We are going to calculate the dimension of $X_{\mu}\left(\varepsilon^{\nu}\right)$, using the obvious fact that $X_{\mu}\left(\varepsilon^{\nu}\right)$ is preserved by the action of $A(F)$.

\subsection{Topology on $X$}

We view $X$ as an ind-scheme in the usual way. Each $K$-orbit on $X$ is finite dimensional, and we denote by $Z_{n}$ the union of all $K$-orbits having dimension less than or equal to $n$. Each $Z_{n}$ is a projective variety, and the increasing family $Z_{0} \subset Z_{1} \subset Z_{2} \subset Z_{3} \subset \cdots$ exhausts $X$. We put the direct limit topology on $X$, so that a subset $Y$ of $X$ is closed (respectively, open) if and only if for all $n$ the intersection $Y \cap Z_{n}$ is closed (respectively, open) in the Zariski topology on $Z_{n}$. Each $Z_{n}$ is closed in $X$. If $Y$ is locally closed in $X$, then each intersection $Y \cap Z_{n}$ is locally closed in $Z_{n}$.

\subsection{Dimensions of locally closed subsets of $X$}

We write $\mathcal{Z}$ for the family of subsets $Z$ of $X$ for which there exists $n$ such that $Z$ is a closed subset of $Z_{n}$. Each $Z \in \mathcal{Z}$ is a projective variety, and the family $\mathcal{Z}$ is stable under the action of $G(L)$. For any locally closed subset $Y$ of $X$ we put

$$
\operatorname{dim}(Y):=\sup \{\operatorname{dim}(Y \cap Z): Z \in \mathcal{Z}\} .
$$

Of course $\operatorname{dim}(Y)$ might be $+\infty$, as happens for example when $Y=X$. Clearly $\operatorname{dim}(g Y)=$ $\operatorname{dim}(Y)$ for all $g \in G(L)$.

\section{4. $U(L)$-orbits on $X$}

For any $\lambda \in X_{*}(A)$ we put $x_{\lambda}:=\varepsilon^{\lambda} x_{0} \in X$. We write $X_{\lambda}$ for the $U(L)$-orbit of $x_{\lambda}$; by the Iwasawa decomposition we have

$$
X=\coprod_{\lambda \in X_{*}(A)} X_{\lambda}
$$

set-theoretically. The $U(L)$-orbits $X_{\lambda}$ are locally closed in $X$. Taking $\lambda=0$ we get $X_{0}=$ $U(L) / U(\mathfrak{o})$. The sets

$$
X_{\leqslant \lambda}:=\coprod_{\lambda^{\prime}: \lambda^{\prime} \leqslant \lambda} X_{\lambda^{\prime}}
$$

are closed in $X$. To prove this, we need to show that for every $Z \in \mathcal{Z}$, the intersection $X_{\leqslant \lambda} \cap Z$ is closed in $Z$. Without loss of generality, it is enough to prove that if $X_{\lambda^{\prime}}$ contains a limit point of $X_{\lambda} \cap Z$, then $\lambda^{\prime} \leqslant \lambda$. For a sufficiently dominant cocharacter $\mu_{0}$, we have $\varepsilon^{\mu_{0}}\left(X_{\lambda} \cap Z\right) \subset$ $U(\mathfrak{o}) \varepsilon^{\mu_{0}+\lambda} x_{0} \subset X^{\mu_{0}+\lambda}$. Thus for such a $\mu_{0}, \varepsilon^{\mu_{0}} X_{\lambda^{\prime}}=X_{\mu_{0}+\lambda^{\prime}}$ meets the closure of $X^{\mu_{0}+\lambda}$. Thus by [25, Lemme 4.2], we see that $\mu_{0}+\lambda^{\prime} \leqslant \mu_{0}+\lambda$, as desired.

4 SÉRIE - TOME $39-2006-\mathrm{N}^{\circ} 3$ 


\subsection{Dimensions of $A(F)$-stable locally closed subsets of $X$}

Let $Y$ be an $A(F)$-stable locally closed subset of $X$. We claim that

$$
\operatorname{dim}(Y)=\operatorname{dim}\left(Y \cap X_{\lambda}\right)
$$

for any $\lambda \in X_{*}(A)$. Clearly $X_{\lambda}=\varepsilon^{\lambda} X_{0}$, and because $Y$ is $A(F)$-stable and hence satisfies $Y=\varepsilon^{\lambda} Y$, we have $Y \cap X_{\lambda}=\varepsilon^{\lambda}\left(Y \cap X_{0}\right)$, so that

$$
\operatorname{dim}\left(Y \cap X_{\lambda}\right)=\operatorname{dim}\left(Y \cap X_{0}\right)
$$

for all $\lambda$. Thus we need only show that

$$
\operatorname{dim}(Y)=\operatorname{dim}\left(Y \cap X_{0}\right)
$$

The inequality $\operatorname{dim}\left(Y \cap X_{0}\right) \leqslant \operatorname{dim}(Y)$ is clear. For the reverse inequality we must show that

$$
\operatorname{dim}(Y \cap Z) \leqslant \operatorname{dim}\left(Y \cap X_{0}\right)
$$

for any $Z \in \mathcal{Z}$. It is easy and well-known (see [25, Lemme 4.2], for instance) that there exists a finite subset $S$ of $X_{*}(A)$ such that $Z$ is contained in

$$
\bigcup_{\lambda \in S} X_{\lambda}
$$

Therefore

$$
\begin{aligned}
\operatorname{dim}(Y \cap Z) & =\sup \left\{\operatorname{dim}\left(Y \cap Z \cap X_{\lambda}\right): \lambda \in S\right\} \\
& \leqslant \sup \left\{\operatorname{dim}\left(Y \cap X_{\lambda}\right): \lambda \in S\right\} .
\end{aligned}
$$

Combining (2.5.5) and (2.5.2), we see that $\operatorname{dim}(Y \cap Z) \leqslant \operatorname{dim}\left(Y \cap X_{0}\right)$, as desired.

Since $X_{\mu}\left(\varepsilon^{\nu}\right)$ is $A(F)$-stable, the remarks above show that its dimension is the same as that of

$$
X_{\mu}\left(\varepsilon^{\nu}\right) \cap X_{0}=\left\{u \in U(L) / U(\mathfrak{o}): u^{-1} \varepsilon^{\nu} \sigma(u) \in K \varepsilon^{\mu} K\right\}
$$

\subsection{Root subgroups of $U$}

For any positive root $\alpha$ we write $U_{\alpha}$ for the root subgroup of $U$ corresponding to $\alpha$. Enumerate the positive roots (in any order) as $\alpha_{1}, \ldots, \alpha_{r}$. Then, as is well-known, the map $\left(u_{1}, \ldots, u_{r}\right) \mapsto u_{1} \cdots u_{r}$ is an isomorphism

$$
\prod_{i=1}^{r} U_{\alpha_{i}} \rightarrow U
$$

of algebraic varieties over $k$. We now fix, for each positive root $\alpha$, an isomorphism $U_{\alpha} \simeq \mathbb{G}_{a}$ over $k$. Thus we may identify $U(L)$ with $L^{r}$ and $U(\mathfrak{o})$ with $\mathfrak{o}^{r}$. 


\subsection{Subgroups $U_{n}$ of $U(\mathfrak{o})$}

For any $n \geqslant 0$ the ring homomorphism $\mathfrak{o} \rightarrow \mathfrak{o} / \varepsilon^{n} \mathfrak{o}$ induces a surjective group homomorphism $U(\mathfrak{o}) \rightarrow U\left(\mathfrak{o} / \varepsilon^{n} \mathfrak{o}\right)$, whose kernel we denote by $U_{n}$. Thus we have a descending chain $U(\mathfrak{o})=$ $U_{0} \supset U_{1} \supset U_{2} \supset \cdots$ of normal subgroups of $U(\mathfrak{o})$. Under our identification of $U(\mathfrak{o})$ with $\mathfrak{o}^{r}$ the subgroup $U_{n}$ becomes identified with $\left(\varepsilon^{n} \mathfrak{o}\right)^{r}$.

\subsection{Subgroups $U(m)$ of $U(L)$}

We now fix a dominant regular coweight $\lambda_{0} \in X_{*}(A)$; thus $\left\langle\alpha, \lambda_{0}\right\rangle>0$ for every positive root $\alpha$ of $A$. Put $a:=\varepsilon^{\lambda_{0}}$, and then for $m \in \mathbb{Z}$ define a subgroup $U(m)$ of $U(L)$ by

$$
U(m):=a^{m} U(\mathfrak{o}) a^{-m} .
$$

There is a chain of inclusions

$$
\cdots \supset U(-2) \supset U(-1) \supset U(0) \supset U(1) \supset U(2) \supset \cdots
$$

and moreover

$$
U(L)=\bigcup_{m \in \mathbb{Z}} U(m) .
$$

Under our identification of $U(L)$ with $L^{r}$, the subgroup $U(m)$ becomes identified with

$$
\prod_{i=1}^{r} \varepsilon^{m\left\langle\alpha_{i}, \lambda_{0}\right\rangle} \mathfrak{o} .
$$

Clearly the filtrations $U_{0} \supset U_{1} \supset U_{2} \supset \cdots$ and $U(0) \supset U(1) \supset U(2) \supset \cdots$ define the same topology on $U(\mathfrak{o})$.

\subsection{Dimensions of locally closed subsets $Y$ of $X_{0}$}

Recall that

$$
X_{0}=U(L) / U(\mathfrak{o}) .
$$

Therefore $X_{0}$ can be written as the increasing union

$$
X_{0}=\bigcup_{m \geqslant 0} U(-m) / U(0)
$$

of its closed subspaces $U(-m) / U(0)$.

Now consider a locally closed subset $Y$ of $X_{0}$. Then $Y$ is locally closed in $X$ and by definition its dimension is $\sup \{\operatorname{dim}(Y \cap Z): Z \in \mathcal{Z}\}$. It is clear that for any $Z \in \mathcal{Z}$ there exists $m \geqslant 0$ such that $Z \cap X_{0} \subset U(-m) / U(0)$. It is equally clear that for any $m \geqslant 0$ there exists $Z \in \mathcal{Z}$ such that $U(-m) / U(0) \subset Z \cap X_{0}$. We conclude that

$$
\operatorname{dim}(Y)=\sup \left\{\operatorname{dim} Y \cap\left(U(-m) / U_{0}\right): m \geqslant 0\right\} .
$$

We of course are particularly interested in the dimension of the locally closed subset

$$
Y_{\mu, \nu}:=X_{\mu}\left(\varepsilon^{\nu}\right) \cap X_{0}=\left\{u \in U(L) / U(\mathfrak{o}): u^{-1} \varepsilon^{\nu} \sigma(u) \in K \varepsilon^{\mu} K\right\},
$$

$4^{\text {e }}$ SÉRIE - TOME $39-2006-\mathrm{N}^{\circ} 3$ 
defined for any coweights $\mu, \nu$ with $\mu$ dominant. In other words

$$
Y_{\mu, \nu}=f_{\nu}^{-1}\left(K \varepsilon^{\mu} K \varepsilon^{-\nu} \cap U(L)\right) / U(\mathfrak{o}),
$$

where $f_{\nu}$ is the map $U(L) \rightarrow U(L)$ defined by $f_{\nu}(u):=u^{-1} \varepsilon^{\nu} \sigma(u) \varepsilon^{-\nu}$.

\subsection{Dimensions for admissible and ind-admissible subsets of $U(L)$}

In view of (2.9.3) we see that it would be useful to introduce a notion of dimension for suitable subsets $V$ of $U(L)$ and then to compute the dimension of $f_{\nu}^{-1} V$ in terms of that of $V$.

For $m, n \geqslant 0$ the quotient $U(-m) / U(n)$ is the set of $\bar{k}$-points of an algebraic variety over $k$. We say that a subset $V$ of $U(-m)$ is admissible if there exist $n \geqslant 0$ and a locally closed subset $V^{\prime}$ of $U(-m) / U(n)$ such that $V$ is the full inverse image of $V^{\prime}$ under $U(-m) \rightarrow U(-m) / U(n)$. We say that a subset $V$ of $U(L)$ is admissible if there exists $m \geqslant 0$ such that $V$ is an admissible subset of $U(-m)$. We say that a subset $V$ of $U(L)$ is ind-admissible if $V \cap U(-m)$ is admissible for all $m \geqslant 0$. Obviously admissible subsets are also ind-admissible.

For any admissible subset $V$ of $U(L)$ we choose $n \geqslant 0$ such that $V$ is preserved by right multiplication by $U(n)$ and then put

$$
\operatorname{dim} V:=\operatorname{dim}(V / U(n))-\operatorname{dim}(U(0) / U(n)) ;
$$

this is clearly independent of the choice of $n$. In this definition we could equally well have used the subgroups $U_{n}$ instead of $U(n)$. Clearly $\operatorname{dim} U(\mathfrak{o})=0$, and of course $\operatorname{dim} V$ can be negative.

For any ind-admissible subset $V$ of $U(L)$ we put

$$
\operatorname{dim} V:=\sup \{\operatorname{dim} V \cap U(-m): m \geqslant 0\} .
$$

Of course $\operatorname{dim}(V)$ can be $+\infty$, as happens in case $V=U(L)$.

\subsection{Warm-up exercise and key proposition}

The following familiar lemma is what makes the next proposition work.

LEMMA 2.11.1. - Let a, b be non-negative integers and consider the group homomorphism $f: \mathfrak{o} \rightarrow \mathfrak{o}$ defined by $f(x):=\varepsilon^{a} \sigma(x)-\varepsilon^{b} x$. Then the image of $f$ is the subgroup $\varepsilon^{c} \mathfrak{o}$, where $c:=\min \{a, b\}$.

Proof. - Clearly it is enough to treat the case in which $c=0$. Then at least one of $a, b$ is zero. If $(a, b) \neq(0,0)$, then $f$ preserves the filtration

$$
\mathfrak{o} \supset \varepsilon \mathfrak{o} \supset \varepsilon^{2} \mathfrak{o} \supset \cdots
$$

of $\mathfrak{o}$ and induces an isomorphism on the associated graded group. Therefore $f$ is bijective in this case.

If $(a, b)=(0,0)$, then $f$ preserves the filtration above and induces on each successive quotient $\bar{k}$ the map $x \mapsto \sigma(x)-x=x^{q}-x$, which is surjective since $\bar{k}$ is algebraically closed. Therefore $f$ maps $\mathfrak{o}$ onto $\mathfrak{o}$.

Now we come to the key proposition. It involves two dominant coweights $\nu$ and $\nu^{\prime}$. Define homomorphisms $\phi, \psi: U(L) \rightarrow U(L)$ by $\phi(u):=\varepsilon^{\nu^{\prime}} u \varepsilon^{-\nu^{\prime}}$ and $\psi(u)=\varepsilon^{\nu} \sigma(u) \varepsilon^{-\nu}$. The dominance of $\nu, \nu^{\prime}$ implies that both $\phi, \psi$ preserve the normal subgroups $U_{n}$ of $U(\mathfrak{o})$ and hence induce homomorphisms $\phi_{n}, \psi_{n}: U\left(\mathfrak{o} / \varepsilon^{n} \mathfrak{o}\right) \rightarrow U\left(\mathfrak{o} / \varepsilon^{n} \mathfrak{o}\right)$. 
We use $\phi, \psi$ to define a right action, denoted $*$, of $U(\mathfrak{o})$ on itself: the action of an element $u \in U(\mathfrak{o})$ upon an element $u^{\prime} \in U(\mathfrak{o})$ is given by $u^{\prime} * u:=\phi(u)^{-1} u^{\prime} \psi(u)$. Similarly, we use $\phi_{n}, \psi_{n}$ to define a right action, again denoted $*$, of $U\left(\mathfrak{o} / \varepsilon^{n} \mathfrak{o}\right)$ on itself: the action of an element $u \in U\left(\mathfrak{o} / \varepsilon^{n} \mathfrak{o}\right)$ upon an element $u^{\prime} \in U\left(\mathfrak{o} / \varepsilon^{n} \mathfrak{o}\right)$ is given by $u^{\prime} * u:=\phi_{n}(u)^{-1} u^{\prime} \psi_{n}(u)$.

We regard $U\left(\mathfrak{o} / \varepsilon^{n} \mathfrak{o}\right)$ as the set of $\bar{k}$-points of an algebraic group over $k$ in the usual way. In particular the underlying variety is simply an affine space of dimension $n \operatorname{dim} U$. In the next proposition the dimensions are those of varieties over $\bar{k}$. In the proposition, and throughout the paper, we write sums over the set of positive roots as $\sum_{\alpha>0}$.

PROPOSITION 2.11.2. - Let $\nu$ and $\nu^{\prime}$ be dominant coweights, and let $n$ be a non-negative integer large enough that

$$
n \geqslant \min \left\{\langle\alpha, \nu\rangle,\left\langle\alpha, \nu^{\prime}\right\rangle\right\}
$$

for every positive root $\alpha$. Then we have:

(1) The codimension of the $U\left(\mathfrak{o} / \varepsilon^{n} \mathfrak{o}\right)$-orbit of $1 \in U\left(\mathfrak{o} / \varepsilon^{n} \mathfrak{o}\right)$ is equal to

$$
\sum_{\alpha>0} \min \left\{\langle\alpha, \nu\rangle,\left\langle\alpha, \nu^{\prime}\right\rangle\right\}
$$

which in turn is equal to the dimension of the stabilizer $S_{n}$ of $1 \in U\left(\mathfrak{o} / \varepsilon^{n} \mathfrak{o}\right)$ in $U\left(\mathfrak{o} / \varepsilon^{n} \mathfrak{o}\right)$. Note that $S_{n}=\left\{u \in U\left(\mathfrak{o} / \varepsilon^{n} \mathfrak{o}\right): \phi_{n}(u)=\psi_{n}(u)\right\}$.

(2) The $U(\mathfrak{o})$-orbit of $1 \in U(\mathfrak{o})$ coincides with the inverse image under $U(\mathfrak{o}) \rightarrow U\left(\mathfrak{o} / \varepsilon^{n} \mathfrak{o}\right)$ of the $U\left(\mathfrak{o} / \varepsilon^{n} \mathfrak{o}\right)$-orbit of $1 \in U\left(\mathfrak{o} / \varepsilon^{n} \mathfrak{o}\right)$. In particular the $U(\mathfrak{o})$-orbit of $1 \in U(\mathfrak{o})$ contains $U_{n}$.

Proof. - To simplify notation we sometimes write $H$ for the group $U\left(\mathfrak{o} / \varepsilon^{n} \mathfrak{o}\right)$. We begin by proving the first statement. The codimension of the $H$-orbit of $1 \in H$ is

$$
\operatorname{dim} H-\operatorname{dim}\left(H / S_{n}\right)=\operatorname{dim} S_{n} .
$$

Under our identification $\mathbb{G}_{a}^{r} \simeq U$, the subgroup $S_{n}$ of $U\left(\mathfrak{o} / \varepsilon^{n} \mathfrak{o}\right)$ goes over to

$$
\prod_{i=1}^{r}\left\{x \in \mathfrak{o} / \varepsilon^{n} \mathfrak{o}: \varepsilon^{\left\langle\alpha_{i}, \nu^{\prime}\right\rangle} x=\varepsilon^{\left\langle\alpha_{i}, \nu\right\rangle} \sigma(x)\right\},
$$

and the dimension of the $i$-th factor in this product is equal to the codimension of the image of the homomorphism $x \mapsto \varepsilon^{\left\langle\alpha_{i}, \nu\right\rangle} \sigma(x)-\varepsilon^{\left\langle\alpha_{i}, \nu^{\prime}\right\rangle} x$ from $\mathfrak{o} / \varepsilon^{n} \mathfrak{o}$ to itself, and by Lemma 2.11.1 (and our hypothesis on $n$ ) this codimension is obviously $\min \left\{\left\langle\alpha_{i}, \nu\right\rangle,\left\langle\alpha_{i}, \nu^{\prime}\right\rangle\right\}$.

Now we prove the second statement. For $u \in U(\mathfrak{o})$ we write $\bar{u}$ for the image of $u$ under $U(\mathfrak{o}) \rightarrow H$. It is clear that the $*$-actions on $U(\mathfrak{o})$ and $H$ are compatible, in the sense that

$$
\overline{u * u^{\prime}}=\bar{u} * \bar{u}^{\prime},
$$

from which it follows that $U(\mathfrak{o}) \rightarrow H$ maps the $U(\mathfrak{o})$-orbit of $1 \in U(\mathfrak{o})$ into the $H$-orbit of $1 \in H$. We must prove that if $u \in U(\mathfrak{o})$ has the property that $\bar{u}$ lies in the $H$-orbit of 1 , then $u$ lies in the $U(\mathfrak{o})$-orbit of 1 . Replacing $u$ by $u * u^{\prime}$ for suitable $u^{\prime} \in U(\mathfrak{o})$, we may assume that $\bar{u}=1$. In other words it remains only to show that any $u \in U_{n}$ lies in the $U(\mathfrak{o})$-orbit of $1 \in U(\mathfrak{o})$. Some care is needed since the $*$-action of $U(\mathfrak{o})$ on itself does not preserve the subgroup $U_{n}$.

Until now we have been working with any ordering $\alpha_{1}, \ldots, \alpha_{r}$ of the positive roots. Now let us order them so that ht $\left(\alpha_{1}\right) \leqslant h t\left(\alpha_{2}\right) \leqslant \cdots \leqslant h t\left(\alpha_{r}\right)$, where $h t(\alpha)$ is the number of simple roots

4 SÉRIE - TOME $39-2006-\mathrm{N}^{\circ} 3$ 
needed in order to write the positive root $\alpha$ as a sum of simple roots. We then get a decreasing chain

$$
U=U[1] \supset U[2] \supset U[3] \supset \cdots \supset U[r+1]=\{1\}
$$

of normal subgroups $U[j]$ of $U$, with $U[j]$ defined as the subgroup consisting of elements whose projections onto the first $j-1$ root subgroups $U_{\alpha_{1}}, \ldots, U_{\alpha_{j-1}}$ are all equal to 1 .

Now return to our element $u \in U_{n}$, and let $u_{1}$ denote the image of $u$ in $U[1](\mathfrak{o}) / U[2](\mathfrak{o})=$ $U_{\alpha_{1}}(\mathfrak{o})$. By Lemma 2.11.1 (and our hypothesis on $n$ ) we may choose $v \in U_{\alpha_{1}}(\mathfrak{o})$ so that $u_{1} * v=1$. It follows that $u * v \in U[2](\mathfrak{o})$. We claim that $u * v \in U_{n}$. For this we must check that $\overline{u * v}=1$, which is clear since

$$
\overline{u * v}=\bar{u} * \bar{v}=1 * \bar{v}=\bar{u}_{1} * \bar{v}=\overline{u_{1} * v}=1 .
$$

Replacing $u$ by $u * v$, we may now assume that $u \in U[2](\mathfrak{o}) \cap U_{n}$. Using $U_{\alpha_{2}}$ the same way we just used $U_{\alpha_{1}}$, we may push $u$ down into $U[3](\mathfrak{o}) \cap U_{n}$. Continuing in this way, we eventually end up with $u \in U[r+1](\mathfrak{o})=\{1\}$, at which point we are done.

\subsection{Formula for the dimension of $f_{\nu, \nu^{\prime}}^{-1} V$}

Now let $\nu, \nu^{\prime} \in X_{*}(A)$ and define a map $f_{\nu, \nu^{\prime}}: U(L) \rightarrow U(L)$ by

$$
f_{\nu, \nu^{\prime}}(u):=\left(\varepsilon^{\nu^{\prime}} u \varepsilon^{-\nu^{\prime}}\right)^{-1} \cdot\left(\varepsilon^{\nu} \sigma(u) \varepsilon^{-\nu}\right)=\varepsilon^{\nu^{\prime}} u^{-1} \varepsilon^{\nu-\nu^{\prime}} \sigma(u) \varepsilon^{-\nu} .
$$

Note that when $\nu^{\prime}=0$, we get back the map $f_{\nu}$ considered earlier. Moreover, when $\nu, \nu^{\prime}$ are both dominant and $u \in U(\mathfrak{o})$, we have $f_{\nu, \nu^{\prime}}(u)=1 * u$, with $*$ denoting the action of $U(\mathfrak{o})$ on itself introduced in Section 2.11.

Now let $\lambda \in X_{*}(A)$. We will also need the conjugation map $c_{\lambda}: U(L) \rightarrow U(L)$ defined by

$$
c_{\lambda}(u):=\varepsilon^{\lambda} u \varepsilon^{-\lambda} .
$$

Proposition 2.12.1. - Let $\lambda, \nu, \nu^{\prime} \in X_{*}(A)$. Then we have:

(1) $f_{\nu, \nu^{\prime}} c_{\lambda}=f_{\lambda+\nu, \lambda+\nu^{\prime}}=c_{\lambda} f_{\nu, \nu^{\prime}}$.

(2) Let $V$ be an admissible (respectively, ind-admissible) subset of $U(L)$. Then $c_{\lambda}^{-1} V$ is admissible (respectively, ind-admissible) and

$$
\operatorname{dim} c_{\lambda}^{-1} V=\operatorname{dim} V+\sum_{\alpha>0}\langle\alpha, \lambda\rangle
$$

(3) Let $V$ be an admissible subset of $U(L)$. Then $f_{\nu, \nu^{\prime}}^{-1} V$ is ind-admissible and

$$
\operatorname{dim} f_{\nu, \nu^{\prime}}^{-1} V=\operatorname{dim} V+\sum_{\alpha>0} \min \left\{\langle\alpha, \nu\rangle,\left\langle\alpha, \nu^{\prime}\right\rangle\right\}
$$

Proof. - The first statement of the proposition is an easy calculation. Next we prove the second statement. Assume that $V$ is an admissible subset of $U(L)$, and pick $n$ big enough that $V$ is stable under right multiplication by $U_{n}$. Since $c_{\lambda}$ induces an isomorphism from the variety $c_{\lambda}^{-1} V / c_{\lambda}^{-1} U_{n}$ to the variety $V / U_{n}$, we see that

$$
\operatorname{dim} c_{\lambda}^{-1} V-\operatorname{dim} c_{\lambda}^{-1} U_{n}=\operatorname{dim} V-\operatorname{dim} U_{n},
$$


from which it follows that it is enough to prove the second statement of the proposition for $V=U_{n}$. Using the root subgroups $U_{\alpha}$, we are reduced to the obvious fact that

$$
\operatorname{dim} \varepsilon^{a} \mathfrak{o} / \varepsilon^{b} \mathfrak{o}=b-a .
$$

Finally we prove the third statement of the proposition. Using the first two parts of the proposition, one sees easily that the third part is true for $\left(\nu, \nu^{\prime}\right)$ if and only if it is true for $\left(\nu+\lambda, \nu^{\prime}+\lambda\right)$. Therefore we may assume without loss of generality that both $\nu, \nu^{\prime}$ are dominant. From now on we abbreviate $f_{\nu, \nu^{\prime}}$ to $f$. Note that the dominance of $\nu, \nu^{\prime}$ implies that $f\left(U_{0}\right) \subset U_{0}$; indeed, for $u \in U_{0}$ we have $f(u)=1 * u$ in the notation of Section 2.11, so that $f\left(U_{0}\right)$ coincides with the $U_{0}$-orbit of $1 \in U_{0}$, something we have analyzed in Proposition 2.11.2. We write $f_{0}$ for the map $f_{0}: U_{0} \rightarrow U_{0}$ obtained by restriction from $f$.

As in Proposition 2.11.2 we now choose a non-negative integer $n_{0}$ large enough that

$$
n_{0} \geqslant \min \left\{\langle\alpha, \nu\rangle,\left\langle\alpha, \nu^{\prime}\right\rangle\right\}
$$

for every positive root $\alpha$. Using the first two parts of Proposition 2.12.1 again, we see that the third part of the proposition is true for $V$ if and only if it is true for $\varepsilon^{\lambda} V \varepsilon^{-\lambda}$. Therefore we may assume without loss of generality that $V \subset U_{n_{0}}$.

Under this assumption we are going to prove that for all $m \geqslant 0$ the intersection $U(-m) \cap$ $f^{-1} V$ is admissible of dimension

$$
\operatorname{dim} V+\sum_{\alpha>0} \min \left\{\langle\alpha, \nu\rangle,\left\langle\alpha, \nu^{\prime}\right\rangle\right\}
$$

which will be enough to prove the proposition.

Recall that $U(-m)=\varepsilon^{-m \lambda_{0}} U_{0} \varepsilon^{m \lambda_{0}}$. Once again using the first two parts of this proposition, we see that

$$
U(-m) \cap f^{-1} V=\varepsilon^{-m \lambda_{0}}\left(U_{0} \cap f^{-1}\left(\varepsilon^{m \lambda_{0}} V \varepsilon^{-m \lambda_{0}}\right)\right) \varepsilon^{m \lambda_{0}}
$$

and that

$$
\operatorname{dim} U(-m) \cap f^{-1} V-\operatorname{dim} V=\operatorname{dim} U_{0} \cap f^{-1}\left(\varepsilon^{m \lambda_{0}} V \varepsilon^{-m \lambda_{0}}\right)-\operatorname{dim} \varepsilon^{m \lambda_{0}} V \varepsilon^{-m \lambda_{0}} .
$$

Since $\varepsilon^{m \lambda_{0}} V \varepsilon^{-m \lambda_{0}}$ is still contained in $U_{n_{0}}$, we may without loss of generality take $m=0$.

Thus we are now reduced to proving that if $V \subset U_{n_{0}}$, then $f_{0}^{-1} V$ is admissible of dimension

$$
\operatorname{dim} V+\sum_{\alpha>0} \min \left\{\langle\alpha, \nu\rangle,\left\langle\alpha, \nu^{\prime}\right\rangle\right\}
$$

Choose $n \geqslant n_{0}$ such that $V$ is invariant under right multiplication by $U_{n}$, and once again put $H:=U_{0} / U_{n}$. Then $V$ is obtained as the inverse image under

$$
p: U_{0} \rightarrow H
$$

of a locally closed subset $\bar{V}$ of $H$ contained in $U_{n_{0}} / U_{n} \subset H$.

Writing $\bar{f}$ for the map $H \rightarrow H$ defined by $\bar{f}(h):=1 * h$, we have $p f_{0}=\bar{f} p$, from which it follows that

$$
f_{0}^{-1} V=p^{-1} \bar{f}^{-1} \bar{V}
$$

$4^{\text {e }}$ SÉRIE - TOME $39-2006-\mathrm{N}^{\circ} 3$ 
In particular $f_{0}^{-1} V$ is admissible of dimension

$$
\operatorname{dim} U_{n}+\operatorname{dim} \bar{f}^{-1} \bar{V}
$$

Recall from before that $S_{n}$ denotes the stabilizer group-scheme of $1 \in H$ for the $*$-action of $H$ on itself. Now the morphism $\bar{f}$ induces an isomorphism from the fpqc quotient $S_{n} \backslash H$ to the $H$-orbit of $1 \in H$ (see Lemma 2.17.2), and by the second part of Proposition 2.11.2, applied to $n_{0}$, we have

$$
\bar{V} \subset U_{n_{0}} / U_{n} \subset \bar{f}(H)
$$

Therefore by Lemma 2.17.2

$$
\operatorname{dim} \bar{f}^{-1} \bar{V}=\operatorname{dim} \bar{V}+\operatorname{dim} S_{n}
$$

from which it follows that

$$
\operatorname{dim} f_{0}^{-1} V=\operatorname{dim} U_{n}+\operatorname{dim} \bar{V}+\operatorname{dim} S_{n}=\operatorname{dim} V+\operatorname{dim} S_{n}
$$

Using the formula for $\operatorname{dim} S_{n}$ given in the first part of Proposition 2.11.2, we see that

$$
\operatorname{dim} f_{0}^{-1} V=\operatorname{dim} V+\sum_{\alpha>0} \min \left\{\langle\alpha, \nu\rangle,\left\langle\alpha, \nu^{\prime}\right\rangle\right\}
$$

as desired.

\subsection{Formula for $\left\langle\rho, \nu-\nu_{\text {dom }}\right\rangle$}

Let $\rho \in X^{*}(A)_{\mathbb{Q}}$ be the half-sum of the positive roots of $A$. Recall that $\nu_{\text {dom }}$ denotes the unique dominant element in the $W$-orbit of $\nu$.

LEMMA 2.13.1. - For any $\nu \in \mathfrak{a}$ there is an equality

$$
\left\langle\rho, \nu-\nu_{\text {dom }}\right\rangle=\sum_{\alpha>0} \min \{\langle\alpha, \nu\rangle, 0\} .
$$

Proof. - The lemma follows immediately from the equations

$$
\langle\rho, \nu\rangle=\sum_{\alpha>0}\langle\alpha, \nu\rangle / 2
$$

and

$$
\left\langle\rho, \nu_{\mathrm{dom}}\right\rangle=\sum_{\alpha>0}|\langle\alpha, \nu\rangle| / 2 .
$$

\subsection{Mirković-Vilonen dimension formula}

Recall that $X_{\nu}$ denotes the $U(L)$-orbit of $x_{\nu}=\varepsilon^{\nu} x_{0}$ in $X$. For dominant $\mu \in X_{*}(A)$ we also consider the $K$-orbit $X^{\mu}:=K x_{\mu}$ of $x_{\mu}$ in $X$. We are interested in the intersection $X^{\mu} \cap X_{\nu}$. We assume that $\nu_{\text {dom }} \leqslant \mu$, since otherwise the intersection is empty (see [1, 4.4.4]). As before $\rho$ is the half-sum of the positive roots. Then we have 
Proposition 2.14.1 (Mirković-Vilonen). - For any dominant $\mu \in X_{*}(A)$ and any $\nu \in$ $X_{*}(A)$ such that $\nu_{\mathrm{dom}} \leqslant \mu$ there is an equality

$$
\operatorname{dim} X^{\mu} \cap X_{\nu}=\langle\rho, \mu+\nu\rangle .
$$

This is proved in [23,22]. Another proof in the present context of finite residue fields is given in Section 5, see Remark 5.5.

We will need the following reformulation of their result.

Proposition 2.14.2. - For any dominant $\mu \in X_{*}(A)$ and any $\nu \in X_{*}(A)$ such that $\nu_{\mathrm{dom}} \leqslant \mu$ there is an equality

$$
\operatorname{dim} K \varepsilon^{\mu} K \varepsilon^{-\nu} \cap U(L)=\langle\rho, \mu-\nu\rangle .
$$

Proof. - The variety $X^{\mu} \cap X_{\nu}$ is isomorphic to the quotient

$$
\left(K \varepsilon^{\mu} K \varepsilon^{-\nu} \cap U(L)\right) / \varepsilon^{\nu} U_{0} \varepsilon^{-\nu},
$$

and $K \varepsilon^{\mu} K \varepsilon^{-\nu} \cap U(L)$ is obviously an admissible subset of $U(L)$. Thus, according to our discussion of dimension theory for admissible subsets of $U(L)$, we have

$$
\operatorname{dim} X^{\mu} \cap X_{\nu}=\operatorname{dim} K \varepsilon^{\mu} K \varepsilon^{-\nu} \cap U(L)-\operatorname{dim} \varepsilon^{\nu} U_{0} \varepsilon^{-\nu} .
$$

From the second part of Proposition 2.12.1 we obtain

$$
\operatorname{dim} \varepsilon^{\nu} U_{0} \varepsilon^{-\nu}=-2\langle\rho, \nu\rangle .
$$

Combining (2.14.1), (2.14.3), (2.14.4), we get the desired result.

\subsection{Main result}

Now we are ready to calculate the dimension of $X_{\mu}\left(\varepsilon^{\nu}\right)$. Recall that $\rho$ denotes the half-sum of the positive roots of $A$.

THEOREM 2.15.1. - Let $\mu, \nu$ be coweights of A. Assume that $\mu$ is dominant and that $\nu_{\text {dom }} \leqslant \mu$. Then

$$
\operatorname{dim} X_{\mu}\left(\varepsilon^{\nu}\right)=\left\langle\rho, \mu-\nu_{\mathrm{dom}}\right\rangle .
$$

Proof. - In Section 2.5 we saw that

$$
\operatorname{dim} X_{\mu}\left(\varepsilon^{\nu}\right)=\operatorname{dim} Y_{\mu, \nu},
$$

where $Y_{\mu, \nu}$ denotes the intersection $X_{\mu}\left(\varepsilon^{\nu}\right) \cap X_{0}$. From (2.9.3) we have

$$
Y_{\mu, \nu}=f_{\nu}^{-1}\left(K \varepsilon^{\mu} K \varepsilon^{-\nu} \cap U(L)\right) / U(\mathfrak{o}) .
$$

Applying Proposition 2.12.1 with $\nu^{\prime}=0$ (so that $f_{\nu, \nu^{\prime}}=f_{\nu}$ ), we see that

$$
\operatorname{dim} Y_{\mu, \nu}=\operatorname{dim} K \varepsilon^{\mu} K \varepsilon^{-\nu} \cap U(L)+\sum_{\alpha>0} \min \{\langle\alpha, \nu\rangle, 0\} .
$$

Using the Mirković-Vilonen dimension formula (Proposition 2.14.2) and Lemma 2.13.1, we obtain

$4^{\text {e }}$ SÉRIE - TOME $39-2006-\mathrm{N}^{\circ} 3$ 


$$
\operatorname{dim} Y_{\mu, \nu}=\langle\rho, \mu-\nu\rangle+\left\langle\rho, \nu-\nu_{\mathrm{dom}}\right\rangle=\left\langle\rho, \mu-\nu_{\mathrm{dom}}\right\rangle
$$

as desired.

\subsection{Remark}

In the last proof we only used Proposition 2.12.1 in the special case $\nu^{\prime}=0$. The reason we took arbitrary $\nu^{\prime}$ in Proposition 2.12.1 was to allow a reduction to the case of dominant coweights in the proof of that proposition. When $\nu^{\prime}=0$ and $\nu$ is dominant, this reduction step is not needed, and since $X_{\mu}\left(\varepsilon^{\nu}\right)$ depends only on the $\sigma$-conjugacy class of $\varepsilon^{\nu}$, hence only on the $W$-orbit of $\nu$, it would have been enough to consider only dominant $\nu$.

Thus one might think it a waste of effort to have formulated and proved Proposition 2.12.1 in the generality that we did. However, later in the paper, when we switch from the affine Grassmannian to the affine flag manifold, we will need Proposition 2.12.1 for $\nu^{\prime}=0$ and arbitrary $\nu$. For non-dominant $\nu$ no simplification occurs when $\nu^{\prime}=0$, and the most natural formulation of Proposition 2.12.1 seems to be the one we have given.

\subsection{Equidimensionality}

Next we will prove that $X_{\mu}\left(\varepsilon^{\nu}\right)$ is equidimensional, in a sense we will make precise below. The equidimensionality follows easily from the corresponding fact about the intersections $X^{\mu} \cap X_{\nu}$ due to Mirković-Vilonen (see Lemma 2.17.4), and from a basic lemma concerning fpqc (or fppf) quotients of algebraic groups by stabilizer subgroup schemes.

We say an ind-admissible set $Y \subset U(L)$ is irreducible if for all $m \geqslant 0$, the intersection $Y \cap U(-m)$ is the full inverse image of an irreducible locally closed subset $\bar{Y} \subset U(-m) / U(n)$, for some $n \geqslant 0$. Note that if $Y \subset U(-m)$ is the full inverse image of a locally closed subset $\bar{Y}_{n} \subset$ $U(-m) / U(n)$, then $\bar{Y}_{n}$ is irreducible if and only if its inverse image $\bar{Y}_{n^{\prime}} \subset U(-m) / U\left(n^{\prime}\right)$ is irreducible for some (equivalently, all) $n^{\prime} \geqslant n$. This holds because the canonical projection $U(-m) / U\left(n^{\prime}\right) \rightarrow U(-m) / U(n)$ can be realized in suitable coordinates as a projection $\mathbb{A}^{a} \times$ $\mathbb{A}^{b} \rightarrow \mathbb{A}^{a}$ for some non-negative integers $a$ and $b$.

We say an ind-admissible set $Y \subset U(L)$ is equidimensional if for each $m \geqslant 0$, the intersection $Y \cap U(-m)$ is the full inverse image of an equidimensional locally closed subvariety of $U(-m) / U(n)$ for some (equivalently, all) sufficiently large $n \geqslant 0$.

Finally, an $A(F)$-stable locally closed subset $Y \subset X$ will be termed equidimensional provided that the full inverse image in $U(L)$ of $Y \cap X_{0} \subset U(L) / U(\mathfrak{o})$ is an equidimensional indadmissible set.

PROPOSITION 2.17.1. - The affine Deligne-Lusztig variety $X_{\mu}\left(\varepsilon^{\nu}\right)$ is equidimensional.

To prove this proposition, we will need a few lemmas. The first lemma will require some basic facts about fppf quotients, see for instance [6, ch. II, III]. To state the first lemma, suppose that $H$ is an algebraic group over $k$, that is, a reduced and irreducible $k$-group scheme of finite type. Suppose $H$ acts (on the left) on a variety, or more generally on a finite-type $k$-scheme $\mathfrak{X}$. For a closed point $x \in \mathfrak{X}(k)$, we let $\mathcal{O}$ denote the $H$-orbit of $x$, a locally-closed subset of $\mathfrak{X}$ which we give the reduced subscheme structure. Let $H_{x} \subset H$ denote the subgroup scheme which stabilizes the point $x$, and let $H / H_{x}$ denote the fppf-sheaf associated to the fppf-presheaf $R \mapsto H(R) / H_{x}(R)$. Finally, let $\mathfrak{p}_{x}: H \rightarrow \mathcal{O}$ be the morphism $h \mapsto h x$.

LEMMA 2.17.2.-

(1) The morphism $\mathfrak{p}_{x}: H \rightarrow \mathcal{O}$ is fppf, hence it is an epimorphism of fppf-sheaves and induces an isomorphism of fppf-sheaves

$$
H / H_{x} \stackrel{\sim}{\longrightarrow} \mathcal{O} .
$$


(2) For every irreducible subset $Y \subset \mathcal{O}$, the inverse image scheme $\mathfrak{p}_{x}^{-1}(Y)$ is a finite union of irreducible subsets $Z$, each having dimension

$$
\operatorname{dim} Z=\operatorname{dim} Y+\operatorname{dim} H_{x} .
$$

Proof. - For part (1) see the proof of [6, III, $\S 3,5.2]$. In fact it is easy to see that $\mathfrak{p}_{x}$ is faithfully flat: It is generically flat since $\mathcal{O}$ is reduced. Translating by elements of $H$, it follows that $\mathfrak{p}_{x}$ is flat. Further, $\mathfrak{p}_{x}$ is surjective by definition. For part (2), we have by [6, III, $\left.\S 3,5.5\right]$ that $\operatorname{dim} \mathcal{O}=\operatorname{dim} H-\operatorname{dim} H_{x}$. Since $H \rightarrow \mathcal{O}$ is a dominant morphism of irreducible varieties, there exists a dense open subset $U \subset \mathcal{O}$ with the following property: for every irreducible set $Y$ which meets $U$ and every irreducible component $W$ of $\mathfrak{p}_{x}^{-1}(Y)$ which meets $\mathfrak{p}_{x}^{-1}(U)$, we have

$$
\operatorname{dim} W-\operatorname{dim} Y=\operatorname{dim} H-\operatorname{dim} \mathcal{O}=\operatorname{dim} H_{x},
$$

see, e.g. [24, I $\S 8$, Thm. 3]. Since $H$ acts transitively on $\mathcal{O}$, the latter is covered by open subsets having the same property, and this implies (2).

LEMMA 2.17.3. - Let $\nu, \nu^{\prime} \in X_{*}(A)$. If $V \subset U(L)$ is an equidimensional ind-admissible set, then so is $f_{\nu, \nu^{\prime}}^{-1}(V)$.

Proof. - We may assume $V$ is irreducible. We may also assume that $V$ is admissible, since the intersection $f_{\nu, \nu^{\prime}}^{-1}(V) \cap U(-m)$ does not change when $V$ is replaced by $V \cap U\left(-m^{\prime}\right)$ for suitably large $m^{\prime}$.

As in the proof of Proposition 2.12.1, we may replace $\left(\nu, \nu^{\prime}\right)$ with $\left(\lambda+\nu, \lambda+\nu^{\prime}\right)$ for a suitably dominant $\lambda$ and thereby reduce to the case where $\nu$ and $\nu^{\prime}$ are dominant and $f=f_{\nu, \nu}$ satisfies $f\left(U_{0}\right) \subset U_{0}$. Also, we may replace $V$ with $\varepsilon^{\lambda} V \varepsilon^{-\lambda}$ and thus we may assume $V \subset U_{n_{0}}$, where $n_{0}$ is chosen as in Proposition 2.12.1. We claim that for any $m \geqslant 0$, the intersection $U(-m) \cap f^{-1} V$ is a union of finitely many irreducible admissible sets all having the same dimension. As in Proposition 2.12.1, it is enough to treat the case $m=0$.

Recall that $H:=U_{0} / U_{n}$ and $f_{0}$ induces the map $\bar{f}: H \rightarrow H$, which is the right action of $H$ on $1 \in H: \bar{f}(h)=1 * h$. Now as before for any $n \geqslant n_{0}$ we have the commutative diagram

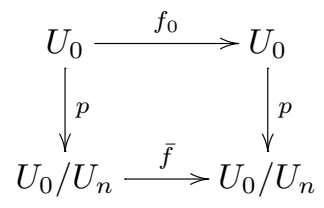

and $\bar{V} \subset U_{n_{0}} / U_{n} \subset \bar{f}(H)$, where all notation is as in Proposition 2.12.1, except that $\bar{V}$ is now an irreducible subset of $U_{0} / U_{n}$.

Since $f_{0}^{-1}(V)=p^{-1} \bar{f}^{-1}(\bar{V})$, we need only show that $\bar{f}^{-1}(\bar{V})$ is the union of finitely many irreducible locally closed subsets of $U_{0} / U_{n}$, all having the same dimension. This follows from Lemma 2.17.2.

The next lemma was established by Mirković and Vilonen as part of their proof of the geometric Satake isomorphism.

Lemma 2.17.4 (Mirković-Vilonen). - Let $\mu$ be G-dominant and let $\nu \in X_{*}(A)$ be an element such that $\nu_{\mathrm{dom}} \leqslant \mu$. Then the variety $X^{\mu} \cap X_{\nu}$ is equidimensional.

Proof. - We shall deduce the lemma from the following result. Let $\mathrm{IC}_{\mu}$ denote the intersection complex on the closure of $X^{\mu}$, normalized in such a way that $\mathrm{IC}_{\mu}[\langle 2 \rho, \mu\rangle](\langle\rho, \mu\rangle)$ is a self-dual

$4^{e}$ SÉRIE - TOME $39-2006-\mathrm{N}^{\circ} 3$ 
perverse sheaf of weight 0 . Then the complex $\mathrm{R}_{c}\left(X_{\nu}, \mathrm{IC}_{\mu}\right)$ is concentrated in cohomological degree $\langle 2 \rho, \mu+\nu\rangle$. This is a part of the geometric Satake isomorphism [23,22], and it was also proved in a direct fashion by Ngô and Polo in [25]. Our goal is simply to take it as an input and show how it implies the equidimensionality statement.

Suppose $X^{\mu} \cap X_{\nu}$ is not equidimensional, and choose an irreducible component $C$ of dimension $d<\langle\rho, \mu+\nu\rangle$. Let $U$ denote the complement in $X^{\mu} \cap X_{\nu}$ of all the other irreducible components. Thus $U$ is open and dense in $C$ and it is also open in $\overline{X^{\mu}} \cap X_{\nu}$. Let $Z$ denote the complement of $U$ in $\overline{X^{\mu}} \cap X_{\nu}$.

Consider the exact sequence

$$
H_{c}^{2 d-1}\left(Z, \mathrm{IC}_{\mu}\right) \rightarrow H_{c}^{2 d}\left(U, \mathrm{IC}_{\mu}\right) \rightarrow H_{c}^{2 d}\left(\overline{X^{\mu}} \cap X_{\nu}, \mathrm{IC}_{\mu}\right) .
$$

The first map is identically zero for weight reasons: the complex $\mathrm{IC}_{\mu}$ has weights $\leqslant 0$, and so by [5], the left-most group has weights $\leqslant 2 d-1$. On the other hand the middle group is pure of weight $2 d$, since $\left.\mathrm{IC}_{\mu}\right|_{U}$ is the constant sheaf. This shows that the non-zero middle group injects into $H_{c}^{2 d}\left(X_{\nu}, \mathrm{IC}_{\mu}\right)$, a contradiction.

Finally, note that Proposition 2.17.1 follows immediately from Lemmas 2.17.3 and 2.17.4 and the following identities we already proved:

$$
\begin{aligned}
X_{\mu}\left(\varepsilon^{\nu}\right) \cap X_{0} & =f_{\nu}^{-1}\left(K \varepsilon^{\mu} K \varepsilon^{-\nu} \cap U(L)\right) / U(\mathfrak{o}), \\
X^{\mu} \cap X_{\nu} & =\left(K \varepsilon^{\mu} K \varepsilon^{-\nu} \cap U(L)\right) / \varepsilon^{\nu} U(\mathfrak{o}) \varepsilon^{-\nu} .
\end{aligned}
$$

\section{Dimension theory for admissible subsets of $L^{n}$}

Let $V$ be a finite dimensional vector space over $L$. We discuss admissibility and dimensions for subsets of $V$. This will be needed in the next section.

\subsection{Lattices}

Recall that a lattice $\Lambda$ in $V$ is a finitely generated o-submodule of $V$ that generates $V$ as vector space. If $V$ is $n$-dimensional, then every lattice in $V$ is free of rank $n$ as o-module. Given two lattices $\Lambda_{1}, \Lambda_{2}$ in $V$, there exist $j, l \in \mathbb{Z}$ such that $\varepsilon^{j} \Lambda_{2} \subset \Lambda_{1} \subset \varepsilon^{l} \Lambda_{2}$, and moreover $\Lambda_{1} \cap \Lambda_{2}$ and $\Lambda_{1}+\Lambda_{2}$ are again lattices.

\subsection{Admissible subsets of $V$}

We say that a subset $Y \subset V$ is admissible if there exist lattices $\Lambda_{2} \subset \Lambda_{1}$ and a Zariski locally closed subset $\bar{Y}$ of the finite dimensional $\bar{k}$-vector space $\Lambda_{1} / \Lambda_{2}$ such that $Y$ is the inverse image under $\Lambda_{1} \rightarrow \Lambda_{1} / \Lambda_{2}$ of $\bar{Y}$; in this situation we say that $Y$ comes from $\bar{Y}$. We say that a subset $Y \subset V$ is ind-admissible if for every lattice $\Lambda$ the intersection $Y \cap \Lambda$ is admissible. Note that a subset $Y$ is ind-admissible if $Y \cap \Lambda_{\alpha}$ is admissible for all $\Lambda_{\alpha}$ in a cofinal family of lattices.

\subsection{Dimension theory for admissible subsets of $V$}

Let $\Lambda$ be a lattice and let $Y$ be an admissible subset of $V$. Choose lattices $\Lambda_{2} \subset \Lambda_{1}$ such that $\Lambda_{2} \subset \Lambda$ and $Y$ comes from $\bar{Y} \subset \Lambda_{1} / \Lambda_{2}$, and put

$$
\operatorname{dim}_{\Lambda} Y:=\operatorname{dim} \bar{Y}-\operatorname{dim} \Lambda / \Lambda_{2}
$$


a number independent of the choice of $\Lambda_{1}, \Lambda_{2}$. This notion of dimension depends of course on $\Lambda$, as the notation indicates. We have normalized things so that $\operatorname{dim} \Lambda=0$. However, for any two admissible subsets $Y_{1}, Y_{2}$ the difference

$$
\operatorname{dim}_{\Lambda} Y_{1}-\operatorname{dim}_{\Lambda} Y_{2}
$$

is independent of $\Lambda$, and so we will permit ourselves to write such differences as simply

$$
\operatorname{dim} Y_{1}-\operatorname{dim} Y_{2}
$$

For any ind-admissible subset $Y \subset V$ we define $\operatorname{dim}_{\Lambda} Y$ to be the supremum of the numbers $\operatorname{dim}_{\Lambda} Y \cap \Lambda_{1}$ as $\Lambda_{1}$ ranges through all lattices.

LEMMA 3.3.1. - Let $g$ be an L-linear automorphism of $V$. Then for any admissible subset $Y \subset V$ the inverse image $g^{-1} Y$ is admissible and

$$
\operatorname{dim} g^{-1} Y-\operatorname{dim} Y=\text { val } \operatorname{det} g .
$$

Proof. - Easy.

\section{Relative dimension of certain morphisms $f: V \rightarrow V$}

This section contains a generalization of Lemma 2.11.1 that will be needed in the next section.

\subsection{Review of $F$-spaces}

Recall that an $F$-space is a pair $(V, \Phi)$, with $V$ a finite dimensional $L$-vector space and $\Phi$ a $\sigma$-linear bijection $V \rightarrow V$; cf. [7,19]. The category of $F$-spaces is an $F$-linear abelian category in which every object is a finite direct sum of simple objects. The isomorphism classes of simple objects are in natural bijection with $\mathbb{Q}$, and a simple $F$-space corresponding to $\lambda \in \mathbb{Q}$ is said to have slope $\lambda$. For $\lambda \in \mathbb{Q}$ we write $V_{\lambda}$ for the sum of all the simple sub- $F$-spaces of $V$ having slope $\lambda$. We then have the slope decomposition

$$
V=\bigoplus_{\lambda \in \mathbb{Q}} V_{\lambda}
$$

Suppose that $V$ is $n$-dimensional. We get an unordered family of $n$ rational numbers by including each $\lambda \in \mathbb{Q}$ in the family with multiplicity equal to $\operatorname{dim} V_{\lambda}$. These $n$ rational numbers are called the slopes of $V$.

Let $\lambda=s / r \in \mathbb{Q}$, with $r \geqslant 1$ and $(r, s)=1$. We get a simple $F$-space of slope $\lambda$ by taking $V=L^{r}$ and $\Phi_{\lambda}\left(a_{1}, \ldots, a_{r}\right)=\left(\varepsilon^{s} \sigma a_{r}, \sigma a_{1}, \ldots, \sigma a_{r-1}\right)$. It follows that for any $F$-space $V$ the number $\lambda \operatorname{dim} V_{\lambda}$ is an integer.

\subsection{Definition of $f: V \rightarrow V$ and $d(V, \Phi)$}

For any $F$-space $(V, \Phi)$ we define an $F$-linear map $f: V \rightarrow V$ by

$$
f(v):=\Phi(v)-v
$$

$4^{\text {e }}$ SÉRIE - TOME $39-2006-\mathrm{N}^{\circ} 3$ 
Moreover we define an integer $d(V, \Phi)$ by

$$
d(V, \Phi):=\sum_{\lambda<0} \lambda \operatorname{dim} V_{\lambda}
$$

LEMMA 4.2.1. - The following statements hold for $V, \Phi, f, d(V, \Phi)$ as above.

(1) The map $f$ is surjective.

(2) For any lattices $\Lambda_{2} \subset \Lambda_{1}$ the group

$$
\left(\Lambda_{1} \cap \operatorname{ker} f\right) /\left(\Lambda_{2} \cap \operatorname{ker} f\right)
$$

is finite.

(3) There exists a lattice $\Lambda_{0}$ such that

(a) $\Lambda_{0}^{\prime}:=f \Lambda_{0}$ is a lattice, and

(b) $\operatorname{dim} \Lambda_{0}-\operatorname{dim} \Lambda_{0}^{\prime}=d(V, \Phi)$.

(4) For any lattice $\Lambda$ there exist lattices $\Lambda_{1}, \Lambda_{2}$ such that $\Lambda_{1} \subset f \Lambda \subset \Lambda_{2}$.

(5) For any lattice $\Lambda^{\prime}$ the inverse image $f^{-1} \Lambda^{\prime}$ contains a lattice and is ind-admissible.

(6) If $\Lambda, \Lambda^{\prime}$ are lattices such that $f \Lambda \supset \Lambda^{\prime}$, then

$$
\operatorname{dim} \Lambda \cap f^{-1} \Lambda^{\prime}-\operatorname{dim} \Lambda^{\prime}=d(V, \Phi) .
$$

(7) For any lattice $\Lambda^{\prime}$ there is an equality

$$
\operatorname{dim} f^{-1} \Lambda^{\prime}-\operatorname{dim} \Lambda^{\prime}=d(V, \Phi)
$$

Proof. - We begin by proving the first three parts of the lemma for the simple $F$-spaces $\left(L^{r}, \Phi_{\lambda}\right)$ described above. Recall that $\lambda=s / r$ with $(r, s)=1$. We consider three cases.

First suppose that $s>0$. Then $f$ preserves the standard filtration $\varepsilon^{n} \mathfrak{o}^{r}$ on $L^{r}$, and induces the obviously bijective map

$$
\bar{f}\left(a_{1}, \ldots, a_{r}\right)=\left(-a_{1}, \sigma a_{1}-a_{2}, \ldots, \sigma a_{r-1}-a_{r}\right)
$$

on each successive quotient $\bar{k}^{r}$. Therefore $f$ is bijective and $f\left(\mathfrak{o}^{r}\right)=\mathfrak{o}^{r}$. In this case (1) and (2) are clear, and (3) holds for $\Lambda_{0}=\mathfrak{o}^{r}$.

Next suppose that $s=0$. Then $r=1$ and we are once again looking at $f: L \rightarrow L$ defined by $f(x)=\sigma(x)-x$. From previous work we know that $f(\mathfrak{o})=\mathfrak{o}$ and hence (since $f$ commutes with multiplication by $\left.\varepsilon^{a}\right)$ that $f\left(\varepsilon^{a} \mathfrak{o}\right)=\varepsilon^{a} \mathfrak{o}$ for all $a \in \mathbb{Z}$. Therefore $f$ is surjective. Its kernel is obviously $F$. So (1) and (2) are clear and (3) holds for $\Lambda_{0}=\mathfrak{o}$.

Finally suppose that $s<0$. Put $\Lambda_{0}=\mathfrak{o}^{r}$ and $\Lambda_{0}^{\prime}=\varepsilon^{s} \mathfrak{o} \oplus \mathfrak{o}^{r-1}$. It is then clear that

$$
f \Lambda_{0} \subset \Lambda_{0}^{\prime}
$$

Again using that $f$ commutes with multiplication by powers of $\varepsilon$, we see that

$$
f\left(\varepsilon^{a} \Lambda_{0}\right) \subset \varepsilon^{a} \Lambda_{0}^{\prime}
$$

for all $a \in \mathbb{Z}$. Thus $f$ induces a map from the associated graded group for the filtration $\varepsilon^{a} \Lambda_{0}$ to the one for the filtration $\varepsilon^{a} \Lambda_{0}^{\prime}$. All successive quotients for both filtrations can be identified with 
$\bar{k}^{r}$ in an obvious way, and when this is done the map induced by $f$ on the successive quotients is always the obviously bijective map

$$
\bar{f}\left(a_{1}, \ldots, a_{r}\right)=\left(\sigma a_{r}, \sigma a_{1}-a_{2}, \ldots, \sigma a_{r-1}-a_{r}\right) .
$$

We conclude that $f$ is bijective and that $f \Lambda_{0}=\Lambda_{0}^{\prime}$. In this case (1) and (2) are clear and (3) holds for the lattice $\Lambda_{0}$ we have chosen, since $\operatorname{dim} \Lambda_{0}-\operatorname{dim} \Lambda_{0}^{\prime}=s=d(V, \Phi)$.

Next we prove the first three parts of the lemma in general. Write $V$ as a direct sum of simple $F$-spaces. Then $f$ decomposes accordingly as a direct sum. Since each summand of $f$ is surjective, so is $f$. As for (2) we are free to enlarge $\Lambda_{1}$ and shrink $\Lambda_{2}$, so we may assume that both $\Lambda_{1}$ and $\Lambda_{2}$ are direct sums of lattices in the simple summands of $V$, which allows us to reduce to the simple case that has already been treated. To prove (3) we note that we can find a suitable $\Lambda_{0}$ by taking a direct sum of suitable lattices in the simple summands.

Now we are going to deduce the remaining parts of lemma from the three parts we have already proved. We will no longer need to reduce to the simple case. We begin by choosing a lattice $\Lambda_{0}$ as in (3). In particular $\Lambda_{0}^{\prime}:=f \Lambda_{0}$ is a lattice. To see that (4) is true we pick $a, b$ such that

$$
\varepsilon^{a} \Lambda_{0} \subset \Lambda \subset \varepsilon^{b} \Lambda_{0}
$$

Then

$$
\varepsilon^{a} \Lambda_{0}^{\prime} \subset f \Lambda \subset \varepsilon^{b} \Lambda_{0}^{\prime} .
$$

Next we prove (5). Pick $j$ such that $\varepsilon^{j} \Lambda_{0}^{\prime} \subset \Lambda^{\prime}$. Then $f^{-1} \Lambda^{\prime}$ contains the lattice $\varepsilon^{j} \Lambda_{0}$. To show that $f^{-1} \Lambda^{\prime}$ is ind-admissible it is enough to show that for $l \geqslant 0$ the set $Y_{l}:=\varepsilon^{j-l} \Lambda_{0} \cap f^{-1} \Lambda^{\prime}$ is admissible. This is clear since $Y_{l}$ comes from the closed subset $\bar{Y}_{l}$ of $\varepsilon^{j-l} \Lambda_{0} / \varepsilon^{j} \Lambda_{0}$ obtained as the kernel of the homomorphism

$$
\varepsilon^{j-l} \Lambda_{0} / \varepsilon^{j} \Lambda_{0} \rightarrow \varepsilon^{-l} \Lambda^{\prime} / \Lambda^{\prime}
$$

induced by $f$.

Now we prove the key statement (6). Let $\mathcal{L}$ be the set of pairs $\left(\Lambda, \Lambda^{\prime}\right)$ of lattices such that $f \Lambda \supset \Lambda^{\prime}$. For any pair $\left(\Lambda, \Lambda^{\prime}\right) \in \mathcal{L}$ we put

$$
d\left(\Lambda, \Lambda^{\prime}\right):=\operatorname{dim} \Lambda \cap f^{-1} \Lambda^{\prime}-\operatorname{dim} \Lambda^{\prime} .
$$

We must show that $d\left(\Lambda, \Lambda^{\prime}\right)=d(V, \Phi)$ for all $\left(\Lambda, \Lambda^{\prime}\right) \in \mathcal{L}$. Since we chose $\Lambda_{0}$ so as to satisfy (3), we see that $\left(\Lambda_{0}, \Lambda_{0}^{\prime}\right) \in \mathcal{L}$ and $d\left(\Lambda_{0}, \Lambda_{0}^{\prime}\right)=d(V, \Phi)$. Thus it is enough to show that

$$
d\left(\Lambda, \Lambda^{\prime}\right)=d\left(\Lambda_{0}, \Lambda_{0}^{\prime}\right)
$$

In fact we claim that for any $j \leqslant 0$ such that $\Lambda \subset \varepsilon^{j} \Lambda_{0}$ there is a chain of equalities

$$
d\left(\Lambda, \Lambda^{\prime}\right)=d\left(\varepsilon^{j} \Lambda_{0}, \Lambda^{\prime}\right)=d\left(\varepsilon^{j} \Lambda_{0}, \Lambda^{\prime} \cap \Lambda_{0}^{\prime}\right)=d\left(\varepsilon^{j} \Lambda_{0}, \Lambda_{0}^{\prime}\right)=d\left(\Lambda_{0}, \Lambda_{0}^{\prime}\right) .
$$

Each of these equalities is a consequence of one of the following two statements.

Statement 1. - If $\left(\Lambda, \Lambda^{\prime}\right) \in \mathcal{L}$ and $\Lambda^{\prime \prime} \subset \Lambda^{\prime}$, then $\left(\Lambda, \Lambda^{\prime \prime}\right) \in \mathcal{L}$ and $d\left(\Lambda, \Lambda^{\prime}\right)=d\left(\Lambda, \Lambda^{\prime \prime}\right)$.

Statement 2. - If $\left(\Lambda, \Lambda^{\prime}\right) \in \mathcal{L}$ and $\Lambda \subset \Lambda_{1}$, then $\left(\Lambda_{1}, \Lambda^{\prime}\right) \in \mathcal{L}$ and $d\left(\Lambda, \Lambda^{\prime}\right)=d\left(\Lambda_{1}, \Lambda^{\prime}\right)$.

$4^{\mathrm{e}}$ SÉRIE - TOME $39-2006-\mathrm{N}^{\circ} 3$ 
Statement 1 follows from the fact that $f$ induces an isomorphism

$$
\frac{\Lambda \cap f^{-1} \Lambda^{\prime}}{\Lambda \cap f^{-1} \Lambda^{\prime \prime}} \rightarrow \frac{\Lambda^{\prime}}{\Lambda^{\prime \prime}}
$$

Statement 2 follows from the fact that

$$
\frac{\Lambda_{1} \cap \operatorname{ker} f}{\Lambda \cap \operatorname{ker} f} \simeq \frac{\Lambda_{1} \cap f^{-1} \Lambda^{\prime}}{\Lambda \cap f^{-1} \Lambda^{\prime}},
$$

which implies (by part (2) of this lemma) that $\Lambda_{1} \cap f^{-1} \Lambda^{\prime}$ is a finite union of cosets of $\Lambda \cap f^{-1} \Lambda^{\prime}$.

Part (7) of the lemma follows immediately from part (6).

Now we come to the key proposition. Let $V, V^{\prime}$ be finite dimensional $L$-vector spaces of the same dimension. Let $\phi: V \rightarrow V^{\prime}$ be an $L$-linear bijection and let $\psi: V \rightarrow V^{\prime}$ be a $\sigma$-linear bijection. Put $f:=\psi-\phi$, an $F$-linear map $V \rightarrow V^{\prime}$. Since $\left(V, \phi^{-1} \psi\right)$ is an $F$-space, it makes sense to put $d:=d\left(V, \phi^{-1} \psi\right)$.

In the proposition below we will be considering the kernel ker $\bar{f}$ of a certain homomorphism $\bar{f}$ of algebraic groups over $\bar{k}$. We take this kernel in the naive sense, that is to say, we take the reduced subscheme structure on the closed set $\operatorname{ker} \bar{f}$. We then write $(\operatorname{ker} \bar{f})^{0}$ for the identity component of $\operatorname{ker} \bar{f}$.

PROPOSITION 4.2.2. - For any lattice $\Lambda$ in $V$ there exist a lattice $\Lambda^{\prime}$ in $V^{\prime}$ and a non-negative integer $j$ such that

$$
\varepsilon^{j} \Lambda^{\prime} \subset f \Lambda \subset \Lambda^{\prime}
$$

For any such $\Lambda^{\prime}, j$, and for any $l \geqslant j$ we consider the homomorphism

$$
\bar{f}: \Lambda / \varepsilon^{l} \Lambda \rightarrow \Lambda^{\prime} / \varepsilon^{l} \Lambda^{\prime}
$$

induced by $f$. Then

(1) $\operatorname{im} \bar{f} \supset \varepsilon^{j} \Lambda^{\prime} / \varepsilon^{l} \Lambda^{\prime}$.

(2) $\operatorname{dim} \operatorname{ker} \bar{f}=d+\operatorname{dim} \phi^{-1} \Lambda^{\prime}-\operatorname{dim} \Lambda$.

(3) $(\operatorname{ker} \bar{f})^{0} \subset \varepsilon^{l-j} \Lambda / \varepsilon^{l} \Lambda$.

Proof. - We may use $\phi$ to identify $V^{\prime}$ with $V$. Thus it is enough to prove this proposition when $V^{\prime}=V$ and $\phi=\operatorname{id}_{V}$. Thus $(V, \psi)$ is an $F$-space and $f$ now has the familiar form $\psi$-id.

The existence of $\Lambda^{\prime}, j$ is a consequence of part (4) of Lemma 4.2.1. Part (1) of the lemma follows from our assumption that $\varepsilon^{j} \Lambda^{\prime} \subset f \Lambda$. To prove (2) we note that

$$
\operatorname{ker} \bar{f}=\frac{\Lambda \cap f^{-1}\left(\varepsilon^{l} \Lambda^{\prime}\right)}{\varepsilon^{l} \Lambda},
$$

a set having dimension $d+\operatorname{dim} \Lambda^{\prime}-\operatorname{dim} \Lambda$ by part (6) of Lemma 4.2.1, which can be used since $f \Lambda \supset \varepsilon^{l} \Lambda^{\prime}$.

To prove (3) it is enough to show that

$$
(\operatorname{ker} \bar{f}) \cap\left(\varepsilon^{l-j} \Lambda / \varepsilon^{l} \Lambda\right)
$$

has the same dimension as $\operatorname{ker} \bar{f}$. For this we note that

$$
(\operatorname{ker} \bar{f}) \cap\left(\varepsilon^{l-j} \Lambda / \varepsilon^{l} \Lambda\right)=\frac{\varepsilon^{l-j} \Lambda \cap f^{-1}\left(\varepsilon^{l} \Lambda^{\prime}\right)}{\varepsilon^{l} \Lambda},
$$


a set again having dimension $d+\operatorname{dim} \Lambda^{\prime}-\operatorname{dim} \Lambda$ by part (6) of Lemma 4.2.1, which can be used since $f\left(\varepsilon^{l-j} \Lambda\right) \supset \varepsilon^{l} \Lambda^{\prime}$.

\section{Reduction step for more general elements $b$}

So far we have treated only elements $b \in A(L)$. Now we will prove Rapoport's conjectural formula for $\operatorname{dim} X_{\mu}^{G}(b)$ assuming its validity for basic elements.

\subsection{Notation related to $P=M N$}

We continue with $G \supset B=A U$ and $W$ as in the introduction. In addition we consider a parabolic subgroup $P=M N$ containing $B$. Here $N$ is the unipotent radical of $P$, and $M$ is the unique Levi factor of $P$ that contains $A$. Put $B_{M}:=B \cap M$ and $U_{M}:=U \cap M$, so that $B_{M}=A U_{M}$ is a Borel subgroup of $M$ containing $A$. We write $W_{M}$ for the Weyl group of $A$ in $M$ and view $W_{M}$ as a subgroup of $W$.

We write $A_{M}$ for the identity component of the center of $M$. Then $A_{M}$ is a subtorus of $A$, and $X_{*}\left(A_{M}\right)$ is a subgroup of $X_{*}(A)$. Put $\mathfrak{a}_{M}:=X_{*}\left(A_{M}\right)_{\mathbb{R}}$, a linear subspace of $\mathfrak{a}$.

Let $R_{N}$ denote the set of roots of $A$ in $\operatorname{Lie}(N)$. We write $\rho_{N} \in X^{*}(A)_{\mathbb{Q}}$ for the half-sum of the roots in $R_{N}$. Thus $\rho=\rho_{M}+\rho_{N}$, where $\rho$ (respectively, $\rho_{M}$ ) is the half-sum of the roots of $A$ in $\operatorname{Lie}(U)$ (respectively, $\operatorname{Lie}\left(U_{M}\right)$ ).

For a coweight $\mu$ of $A$ we will need to distinguish between the two relevant notions of dominance. We say that $\mu$ is $G$-dominant (respectively, $M$-dominant) if $\langle\alpha, \mu\rangle \geqslant 0$ for every root $\alpha$ of $A$ in $\operatorname{Lie}(U)$ (respectively, $\operatorname{Lie}\left(U_{M}\right)$ ).

Similarly we need to distinguish between the two relevant partial orders on $X_{*}(A)$. When $\mu-\nu$ is a non-negative integral linear combination of simple coroots for $G$ (respectively, $M$ ), we write $\nu \leqslant \mu$ (respectively, $\nu \underset{M}{\leqslant} \mu$ ).

Recall from the introduction the canonical surjections $p_{G}: X_{*}(A) \rightarrow \Lambda_{G}$ and $\eta_{G}: X^{G}=$ $G(L) / K \rightarrow \Lambda_{G}$. We apply these definitions to $M$ as well as $G$, obtaining $p_{M}: X_{*}(A) \rightarrow \Lambda_{M}$ and

$$
\eta_{M}: X^{M}=M(L) / K_{M} \rightarrow \Lambda_{M},
$$

where $K_{M}$ denotes $M(\mathfrak{o})$. There is an obvious embedding $X^{M} \hookrightarrow X^{G}$. In particular we view $x_{0}$ as the base-point for $X^{M}$ as well as for $X^{G}$.

Note that the composition $X_{*}\left(A_{M}\right) \hookrightarrow X_{*}(A) \rightarrow \Lambda_{M}$ is injective and identifies $X_{*}\left(A_{M}\right)$ with a subgroup of finite index in $\Lambda_{M}$, so that $\mathfrak{a}_{M}$ can also be identified with $\Lambda_{M} \otimes_{\mathbb{Z}} \mathbb{R}$; in this way we obtain a canonical homomorphism

$$
\Lambda_{M} \rightarrow \mathfrak{a}_{M}
$$

\subsection{Dimensions for admissible and ind-admissible subsets of $N(L)$}

In Section 2.8 we defined subgroups

$$
\cdots \supset U(-2) \supset U(-1) \supset U(0) \supset U(1) \supset U(2) \supset \cdots
$$

of $U(L)$, and in Section 2.10 we used these subgroups to define (ind)-admissible subsets of $U(L)$, as well as dimensions of such sets.

For $m \in \mathbb{Z}$ we now put

$$
N(m):=N(L) \cap U(m)
$$

$4^{\text {e }}$ SÉRIE - TOME $39-2006-\mathrm{N}^{\circ} 3$ 
and define (ind)-admissibility and dimensions for subsets of $N(L)$ just as we did for $U(L)$, using the subgroups $N(m)$ in place of $U(m)$.

\subsection{Relative dimension of $f_{m}$}

As before, for $\nu \in \mathfrak{a}$ we write $\nu_{\text {dom }}$ for the unique $G$-dominant element in the $W$-orbit of $\nu$.

Proposition 5.3.1. - Let $m \in M(L)$ and let $\nu$ be the $M$-dominant element of $\mathfrak{a}$ defined as the Newton point of the $\sigma$-conjugacy class of $m$ in $M(L)$. Define a map $f_{m}: N(L) \rightarrow N(L)$ by $f_{m}(n)=n^{-1} m \sigma(n) m^{-1}$. Let $Y$ be an admissible subset of $N(L)$. Then $f_{m}^{-1} Y$ is ind-admissible and

$$
\begin{aligned}
\operatorname{dim} f_{m}^{-1} Y-\operatorname{dim} Y & =\sum_{\alpha \in R_{N}} \min \{\langle\alpha, \nu\rangle, 0\} \\
& =\left\langle\rho, \nu-\nu_{\mathrm{dom}}\right\rangle .
\end{aligned}
$$

Moreover $f_{m}$ is surjective.

Proof. - It would be awkward to prove this proposition as it stands, since conjugation by $m$ need not preserve $N(\mathfrak{o})$. Therefore we are going to reformulate the proposition in a way that will make it easier to prove.

For $m_{1}, m_{2} \in M(L)$ we now define a map $f_{m_{1}, m_{2}}: N(L) \rightarrow N(L)$ by

$$
f_{m_{1}, m_{2}}(n):=m_{1} n^{-1} m_{1}^{-1} \cdot m_{2} \sigma(n) m_{2}^{-1},
$$

which gives back $f_{m}$ when $\left(m_{1}, m_{2}\right)=(1, m)$. Put $\mathfrak{n}:=\operatorname{Lie} N$. For any $m \in M(L)$ we denote by $\operatorname{Ad}_{\mathfrak{n}}(m)$ the adjoint action of $m$ on $\mathfrak{n}(L)$. Now $\operatorname{Ad}_{\mathfrak{n}}\left(m_{1}\right)^{-1} \operatorname{Ad}_{\mathfrak{n}}\left(m_{2}\right) \sigma$ is a $\sigma$-linear bijection from $\mathfrak{n}(L)$ to itself, so $\left(\mathfrak{n}(L), \operatorname{Ad}_{\mathfrak{n}}\left(m_{1}\right)^{-1} \operatorname{Ad}_{\mathfrak{n}}\left(m_{2}\right) \sigma\right)$ is an $F$-space, and we may put

$$
d\left(m_{1}, m_{2}\right):=d\left(\mathfrak{n}(L), \operatorname{Ad}_{\mathfrak{n}}\left(m_{1}\right)^{-1} \operatorname{Ad}_{\mathfrak{n}}\left(m_{2}\right) \sigma\right)+\operatorname{val} \operatorname{det} \operatorname{Ad}_{\mathfrak{n}}\left(m_{1}\right) .
$$

Note that

$$
\begin{aligned}
d(1, m) & =d\left(\mathfrak{n}(L), \operatorname{Ad}_{\mathfrak{n}}(m) \sigma\right) \\
& =\sum_{\alpha \in R_{N}} \min \{\langle\alpha, \nu\rangle, 0\}
\end{aligned}
$$

since by its very definition the Newton point $\nu$ of $m$ has the property that the slopes of the $F$-space $\left(\mathfrak{n}(L), \operatorname{Ad}_{\mathfrak{n}}(m) \sigma\right)$ are the numbers $\langle\alpha, \nu\rangle$ for $\alpha \in R_{N}$. Moreover it follows from Lemma 2.13.1 that

$$
\sum_{\alpha \in R_{N}} \min \{\langle\alpha, \nu\rangle, 0\}=\left\langle\rho, \nu-\nu_{\mathrm{dom}}\right\rangle,
$$

since $\langle\alpha, \nu\rangle \geqslant 0$ for all positive roots $\alpha$ in $M$. Therefore the next proposition does indeed generalize the previous one, and it will be enough to prove it.

Proposition 5.3.2. - The map $f_{m_{1}, m_{2}}$ is surjective. Moreover, for any admissible subset $Y$ of $N(L)$ the inverse image $f_{m_{1}, m_{2}}^{-1} Y$ is ind-admissible and

$$
\operatorname{dim} f_{m_{1}, m_{2}}^{-1} Y-\operatorname{dim} Y=d\left(m_{1}, m_{2}\right) .
$$


Proof. - For any $m \in M(L)$ we have

$$
f_{m m_{1}, m m_{2}}=c_{m} \circ f_{m_{1}, m_{2}},
$$

where $c_{m}: N(L) \rightarrow N(L)$ denotes the conjugation map $n \mapsto m n m^{-1}$. It is easy to see that for any admissible subset $Y$ of $N(L)$ the inverse image $c_{m}^{-1} Y$ is admissible, and that

$$
\operatorname{dim} c_{m}^{-1} Y-\operatorname{dim} Y=\operatorname{val} \operatorname{det} \operatorname{Ad}_{\mathfrak{n}}(m) .
$$

Therefore the proposition is true for $m_{1}, m_{2}$ if and only if it is true for $m m_{1}, m m_{2}$. So this proposition is not more general than the previous one in any serious way, but it is more convenient to prove, since we are free to improve the given pair $\left(m_{1}, m_{2}\right)$ as follows.

Let $M(L)_{+}$denote the union of all $K_{M}$-double cosets $K_{M} \varepsilon^{\mu_{M}} K_{M}$ as $\mu_{M}$ ranges over all $M$-dominant coweights such that $\left\langle\alpha, \mu_{M}\right\rangle \geqslant 0$ for all $\alpha \in R_{N}$.

What is the point of this definition? For any algebraic group $I$ over $k$ and any $j \geqslant 0$ the kernel of the homomorphism $I(\mathfrak{o}) \rightarrow I\left(\mathfrak{o} / \varepsilon^{j} \mathfrak{o}\right)$ is a normal subgroup of $I(\mathfrak{o})$ that we will denote by $I_{j}$. Thus

$$
I(\mathfrak{o})=I_{0} \supset I_{1} \supset I_{2} \supset \cdots
$$

with $I_{0} / I_{j}=I\left(\mathfrak{o} / \varepsilon^{j} \mathfrak{o}\right)$. Applying this definition to $N$ (later we shall apply it to some other groups), we get normal subgroups $N_{j}$ of $N(\mathfrak{o})$, and the point is that for any $m \in M(L)_{+}$and $j \geqslant 0$

$$
m N_{j} m^{-1} \subset N_{j}
$$

There exists $a \in A_{M}(L)$ such that $a m_{1}, a m_{2} \in M(L)_{+}$. Replacing $\left(m_{1}, m_{2}\right)$ by $\left(a m_{1}, a m_{2}\right)$, we see that it is enough to prove the proposition under the additional assumption that $m_{1}, m_{2} \in$ $M(L)_{+}$, an assumption we will make from now on.

Define homomorphisms $\phi, \psi: N(L) \rightarrow N(L)$ by $\phi(n):=m_{1} n m_{1}^{-1}$ and $\psi(n):=$ $m_{2} \sigma(n) m_{2}^{-1}$. Note that $\phi\left(N_{j}\right) \subset N_{j}$ and $\psi\left(N_{j}\right) \subset N_{j}$ for all $j \geqslant 0$. Thus we may define a right action of $N(\mathfrak{o})$ on itself, called the $*$-action, with $n \in N(\mathfrak{o})$ acting on $n^{\prime} \in N(\mathfrak{o})$ by

$$
n^{\prime} * n:=\phi(n)^{-1} n^{\prime} \psi(n) .
$$

The homomorphisms

$$
\phi_{j}, \psi_{j}: N_{0} / N_{j} \rightarrow N_{0} / N_{j}
$$

induced by $\phi, \psi$ respectively allow us to define a right $*$-action of $N_{0} / N_{j}$ on itself by

$$
n^{\prime} * n:=\phi_{j}(n)^{-1} n^{\prime} \psi_{j}(n)
$$

Denoting by $n \mapsto \bar{n}$ the canonical surjection $N_{0} \rightarrow N_{0} / N_{j}$, we have the obvious compatibility

$$
\overline{n^{\prime} * n}=\bar{n}^{\prime} * \bar{n} .
$$

We need to linearize our problem by means of a suitable filtration

$$
N=N[1] \supset N[2] \supset \cdots
$$

of $N$ by normal subgroups of $P$ such that each successive quotient

$$
N\langle i\rangle:=N[i] / N[i+1]
$$

$4^{\text {e }}$ SÉRIE - TOME $39-2006-\mathrm{N}^{\circ} 3$ 
is abelian. To this end we introduce the following coweight $\delta_{N}$ of the quotient $A / Z$ of $A$ by the center $Z$ of $G$. In $X_{*}(A / Z)$ we have the $\mathbb{Z}$-basis of fundamental coweights $\varpi_{\alpha}$, one for each simple root $\alpha$ of $A$. Define the coweight $\delta_{N}$ to be the sum of the fundamental coweights $\varpi_{\alpha}$ with $\alpha$ ranging through the simple roots of $A$ that occur in $\mathfrak{n}=\operatorname{Lie} N$. Then for $i \geqslant 1$ we let $N[i]$ be the product of all root subgroups $U_{\alpha}$ for which $\left\langle\alpha, \delta_{N}\right\rangle \geqslant i$.

Put $\mathfrak{n}[i]:=\operatorname{Lie} N[i]$ and $\mathfrak{n}\langle i\rangle:=\operatorname{Lie} N\langle i\rangle$. Then there is a $P$-equivariant isomorphism

$$
N\langle i\rangle \cong \mathfrak{n}\langle i\rangle
$$

of algebraic groups over $k$, and the action of $P$ on both groups factors through $P \rightarrow M$. (To construct this isomorphism first work with split groups over $\mathbb{Z}$.)

The homomorphisms $\phi, \psi$ preserve the subgroups $N[i](L)$ and therefore induce homomorphisms

$$
\phi\langle i\rangle, \psi\langle i\rangle: N\langle i\rangle(L) \rightarrow N\langle i\rangle(L)
$$

as well, so we get $*$-actions of $N[i](\mathfrak{o})$ and $N\langle i\rangle(\mathfrak{o})$ on themselves. Moreover, under the identification (5.3.4) of $N\langle i\rangle(L)$ with $\mathfrak{n}\langle i\rangle(L)$, the homomorphism $\phi\langle i\rangle$ (respectively, $\psi\langle i\rangle$ ) goes over to the $L$-linear bijection $\operatorname{Ad}_{\mathfrak{n}\langle i\rangle}\left(m_{1}\right)$ (respectively, the $\sigma$-linear bijection $\operatorname{Ad}_{\mathfrak{n}\langle i\rangle}\left(m_{2}\right) \sigma$ ), where $\operatorname{Ad}_{\mathfrak{n}\langle i\rangle}(\cdot)$ denotes the adjoint action of $M$ on $\mathfrak{n}\langle i\rangle=\mathfrak{n}[i] / \mathfrak{n}[i+1]$.

Thus for each $i$ we are in the linear situation considered in Proposition 4.2.2 (with $V=V^{\prime}=$ $\mathfrak{n}\langle i\rangle(L)$ ), and as in that proposition we define an $F$-linear map

$$
f\langle i\rangle: \mathfrak{n}\langle i\rangle(L) \rightarrow \mathfrak{n}\langle i\rangle(L)
$$

by $f\langle i\rangle:=\psi\langle i\rangle-\phi\langle i\rangle$.

Since $m_{1}, m_{2} \in M(L)_{+}$, both $\phi\langle i\rangle$ and $\psi\langle i\rangle$ carry the lattice $\mathfrak{n}\langle i\rangle(\mathfrak{o})$ into itself; therefore $f\langle i\rangle$ does too. By Proposition 4.2.2 there exists $j \geqslant 0$ such that

$$
f\langle i\rangle(\mathfrak{n}\langle i\rangle(\mathfrak{o})) \supset \varepsilon^{j}(\mathfrak{n}\langle i\rangle(\mathfrak{o})) .
$$

Since $\mathfrak{n}\langle i\rangle=0$ for large $i$, we can even choose $j$ so that (5.3.5) holds for all $i$.

We also need to look at this slightly differently. For $a \in A_{M}(F)$ define

$$
c_{a}: N(L) \rightarrow N(L)
$$

by $c_{a}(n):=a n a^{-1}$. The homomorphisms $c_{a}$ preserve the subgroups $N[i](L)$ and hence induce homomorphisms

$$
c_{a}: N\langle i\rangle(L) \rightarrow N\langle i\rangle(L)
$$

For $a \in A_{M}(F)_{+}:=A_{M}(F) \cap M(L)_{+}$we have $c_{a} N_{0} \subset N_{0}, c_{a} N[i]_{0} \subset N[i]_{0}, c_{a} N\langle i\rangle_{0} \subset$ $N\langle i\rangle_{0}$, and it is easy to see that

$$
c_{a}\left(n^{\prime} * n\right)=c_{a}\left(n^{\prime}\right) * c_{a}(n)
$$

for all $n, n^{\prime} \in N_{0}$.

ClaIM 1. - There exists $a \in A_{M}(F)_{+}$such that for any $i \geqslant 1$

$$
c_{a} N[i]_{0} \subset 1 * N[i]_{0} .
$$


Now we prove Claim 1 . We begin by proving the weaker statement that for each $i \geqslant 1$ there exists $a_{i} \in A_{M}(F)_{+}$such that (5.3.6) holds for $a_{i}$ and $i$, and this we prove by descending induction on $i$, starting with any $i$ such that $N[i]$ is trivial, so that the statement we need to prove becomes trivial. Assume the statement is true for $i+1$ and prove it for $i$. Thus we are assuming that there exists $a_{i+1} \in A_{M}(F)_{+}$such that (5.3.6) holds for $a_{i+1}$ and $i+1$.

Applying Proposition 4.2.2 just as we did to find $j$, we see that there exists $a^{\prime} \in A_{M}(F)_{+}$ such that

$$
c_{a^{\prime}} N\langle i\rangle_{0} \subset 1 * N\langle i\rangle_{0} .
$$

We will now check that (5.3.6) holds for $a_{i}:=a^{\prime} a_{i+1}$ and $i$.

Indeed, consider an element $x=c_{a_{i}} n$ with $n \in N[i]_{0}$. The image of $c_{a^{\prime}} n$ under $N[i]_{0} \rightarrow$ $N\langle i\rangle_{0}$ lies in $c_{a^{\prime}} N\langle i\rangle_{0}$, and therefore (5.3.7) guarantees the existence of $n^{\prime} \in N[i]_{0}$ such that $\left(c_{a^{\prime}} n\right) * n^{\prime} \in N[i+1]_{0}$, whence

$$
c_{a_{i+1}}\left(\left(c_{a^{\prime}} n\right) * n^{\prime}\right) \in c_{a_{i+1}} N[i+1]_{0} .
$$

Since (5.3.6) holds for $a_{i+1}$ and $i+1$, there exists $n^{\prime \prime} \in N[i+1]_{0}$ such that

$$
\left(c_{a_{i+1}}\left(\left(c_{a^{\prime}} n\right) * n^{\prime}\right)\right) * n^{\prime \prime}=1 .
$$

The left side of equation (5.3.8) works out to

$$
c_{a_{i}} n *\left(c_{a_{i+1}}\left(n^{\prime}\right) n^{\prime \prime}\right),
$$

showing that $c_{a_{i}} n \in 1 * N[i]_{0}$, as desired. This proves the weaker statement.

To prove the claim itself we first note that for $a, a^{\prime} \in A_{M}(F)_{+}$it is clear that $a a^{\prime} \in A_{M}(F)_{+}$ and that

$$
c_{a a^{\prime}} N[i]_{0}=c_{a}\left(c_{a^{\prime}} N[i]_{0}\right) \subset c_{a} N[i]_{0} .
$$

Therefore if (5.3.6) holds for $a$ and $i$, it also holds for $a a^{\prime}$ and $i$. We already know that for each of the finitely many values of $i$ for which $N[i]$ is non-trivial, we can find an element $a_{i}$ for which (5.3.6) holds for $a_{i}$ and $i$, and taking the product of all these elements $a_{i}$, we get an element $a$ such that (5.3.6) holds for all $i$. This proves the claim.

Now we fix $a \in A_{M}(F)_{+}$as in Claim 1. Choose $j \geqslant 0$ as before, so that (5.3.5) holds for all $i$. For reasons that will soon become apparent, we now consider any $l \geqslant j$ large enough that for all $i \geqslant 1$

$$
N[i]_{l-j} \subset c_{a} N[i]_{0} .
$$

We denote by $H, H[i], H\langle i\rangle$ the groups of $\mathfrak{o} / \varepsilon^{l} \mathfrak{o}$-points of $N, N[i], N\langle i\rangle$ respectively, regarded as algebraic groups over $\bar{k}$. Note that $H=H[1]$ and $H\langle i\rangle=H[i] / H[i+1]$. On $H[i]$, $H\langle i\rangle$ we have descending filtrations $H[i]_{\beta}, H\langle i\rangle_{\beta}$ coming from the powers $\varepsilon^{\beta}$ of $\varepsilon$; more precisely, for $0 \leqslant \beta \leqslant l$ we put

$$
H[i]_{\beta}:=\operatorname{ker}\left[N[i]\left(\mathfrak{o} / \varepsilon^{l} \mathfrak{o}\right) \rightarrow N[i]\left(\mathfrak{o} / \varepsilon^{\beta} \mathfrak{o}\right)\right]
$$

and similarly for $H\langle i\rangle$. The $*$-actions on $N[i](\mathfrak{o})$ and $N\langle i\rangle(\mathfrak{o})$ induce compatible $*$-actions on $H[i]$ and $H\langle i\rangle$.

The homomorphism $c_{a}$ preserves both $N_{0}$ and $N_{l}$ and hence induces a homomorphism $c_{a}: H \rightarrow H$. Since Claim 1 holds for $a$, we see that for any $i \geqslant 1$

$$
c_{a} H[i] \subset 1 * H[i] .
$$

$4^{\text {e }}$ SÉRIE - TOME $39-2006-\mathrm{N}^{\circ} 3$ 
Moreover (5.3.9) implies immediately that

$$
H[i]_{l-j} \subset c_{a} H[i] .
$$

Also note that

$$
H[i] \cap c_{a} H=c_{a} H[i],
$$

as one sees easily from the description of $H$ as a product of copies of $\mathfrak{o} / \varepsilon^{l} \mathfrak{o}$, one for each root in $R_{N}$.

Write $S[i]$ (respectively, $S\langle i\rangle$ ) for the stabilizer, for the $*$-action, of $1 \in H[i]$ in $H[i]$ (respectively, of $1 \in H\langle i\rangle$ in $H\langle i\rangle$ ). Note that $S:=S[1]$ is the stabilizer, for the $*$-action, of $1 \in H$ in $H$. Here we are taking naive stabilizers: put the reduced subscheme structure on the set-theoretic stabilizers.

CLAIM 2. - $\operatorname{dim} S=d\left(m_{1}, m_{2}\right)$.

We begin by proving that

$$
\operatorname{dim} S[i]=\operatorname{dim} S[i+1]+\operatorname{dim} S\langle i\rangle .
$$

For this it is enough to prove that

$$
S\langle i\rangle^{0} \subset \operatorname{im}[S[i] \rightarrow H\langle i\rangle] \subset S\langle i\rangle .
$$

The second inclusion being clear, it is enough to prove the first one. So let $s \in S\langle i\rangle^{0}$. By Proposition 4.2.2 we have

$$
S\langle i\rangle^{0}=(\operatorname{ker} \overline{f\langle i\rangle})^{0} \subset H\langle i\rangle_{l-j} .
$$

Pick $h \in H[i]_{l-j}$ such that $h \mapsto s$. By (5.3.11) there exists $h_{0} \in H[i]$ such that $h=c_{a} h_{0}$. Now $1 * h \mapsto 1 * s=1 \in H\langle i\rangle$, showing that $1 * h \in H[i+1]$. Moreover $1 * h=c_{a}\left(1 * h_{0}\right)$, showing that $1 * h \in c_{a} H$. From (5.3.12) and (5.3.10) we conclude that

$$
1 * h \in c_{a} H[i+1] \subset 1 * H[i+1]
$$

which means that there exists $h_{1} \in H[i+1]$ such that $1 * h h_{1}=1$. Thus $h h_{1} \in S[i]$, and clearly $h h_{1} \mapsto s$, proving that $s$ lies in the image of $S[i]$, as desired. This proves the equality (5.3.13).

It follows from (5.3.13) that

$$
\operatorname{dim} S=\sum_{i \geqslant 1} \operatorname{dim} S\langle i\rangle
$$

Moreover, by part (2) of Proposition 4.2.2

$$
\operatorname{dim} S\langle i\rangle=d\left(\mathfrak{n}\langle i\rangle(L), \phi\langle i\rangle^{-1} \psi\langle i\rangle\right)+\operatorname{dim} \phi\langle i\rangle^{-1}(\mathfrak{n}\langle i\rangle(\mathfrak{o}))-\operatorname{dim} \mathfrak{n}\langle i\rangle(\mathfrak{o}) .
$$

Therefore

$$
\begin{aligned}
\operatorname{dim} S & =d\left(\mathfrak{n}(L), \operatorname{Ad}_{\mathfrak{n}}\left(m_{1}\right)^{-1} \operatorname{Ad}_{\mathfrak{n}}\left(m_{2}\right) \sigma\right)+\operatorname{dim} \operatorname{Ad}_{\mathfrak{n}}\left(m_{1}\right)^{-1} \mathfrak{n}(\mathfrak{o})-\operatorname{dim} \mathfrak{n}(\mathfrak{o}) \\
& =d\left(m_{1}, m_{2}\right),
\end{aligned}
$$

completing the proof of Claim 2.

Now consider the map $f_{0}: N(\mathfrak{o}) \rightarrow N(\mathfrak{o})$ defined by $f_{0}(n):=1 * n$. Equivalently, $f_{0}$ is the restriction of $f_{m_{1}, m_{2}}$ to $N(\mathfrak{o})$. 
Claim 3. - Suppose that $Y$ is an admissible subset of $c_{a} N_{0}$ (with $a$ again as in Claim 1). Then $f_{0}^{-1} Y$ is admissible and

$$
\operatorname{dim} f_{0}^{-1} Y-\operatorname{dim} Y=d\left(m_{1}, m_{2}\right) .
$$

Now we prove Claim 3. The set $Y$ comes from a locally closed subset $\bar{Y}$ of $H=N\left(\mathfrak{o} / \varepsilon^{l} \mathfrak{o}\right)$ for some suitably large $l$. By increasing $l$ we may assume that Claim 2 holds for $l$. We have a commutative diagram

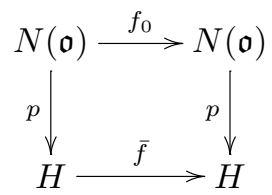

where $p$ is the canonical surjection and $\bar{f}$ is induced by $f_{0}$. By Claim $1 \bar{Y}$ is contained in the image of $\bar{f}$. Every non-empty (reduced) fiber of $\bar{f}$ is isomorphic to the stabilizer group $S$ considered above. Therefore

$$
\operatorname{dim} \bar{f}^{-1} \bar{Y}=\operatorname{dim} \bar{Y}+\operatorname{dim} S=\operatorname{dim} \bar{Y}+d\left(m_{1}, m_{2}\right) .
$$

Since $f_{0}^{-1} Y=p^{-1} \bar{f}^{-1} \bar{Y}$, we see that $f_{0}^{-1} Y$ is admissible and

$$
\operatorname{dim} f_{0}^{-1} Y-\operatorname{dim} Y=d\left(m_{1}, m_{2}\right),
$$

proving Claim 3.

Now we finish the proof. We abbreviate $f_{m_{1}, m_{2}}$ to $f$ and $d\left(m_{1}, m_{2}\right)$ to $d$. Let $Y$ be any admissible subset of $N(L)$.

For any $a^{\prime} \in A_{M}(F)$ it is clear that $f$ commutes with $c_{a^{\prime}}$. Thus it follows from Claim 3 together with (5.3.3) that $f^{-1} Y \cap a^{\prime-1} N(\mathfrak{o}) a^{\prime}$ is admissible of dimension $\operatorname{dim} Y+d$ for all $a^{\prime} \in A_{M}(F)$ such that $a^{\prime} Y a^{\prime-1} \subset a N_{0} a^{-1}$ (with $a$ as before, so that Claims 1 and 3 hold for it).

Pick a coweight $\lambda_{0} \in X_{*}\left(A_{M}\right)$ such that $\left\langle\alpha, \lambda_{0}\right\rangle>0$ for all $\alpha \in R_{N}$, and for $t \in \mathbb{Z}$ put $a_{t}:=\lambda_{0}\left(\varepsilon^{t}\right)$. There exists $t_{0} \geqslant 0$ such that $a_{t} Y a_{t}^{-1} \subset a N_{0} a^{-1}$ for all $t \geqslant t_{0}$. Therefore $f^{-1} Y \cap a_{t}^{-1} N(\mathfrak{o}) a_{t}$ is admissible of dimension $\operatorname{dim} Y+d$ for all $t \geqslant t_{0}$, and since any of the subgroups $N(i)(i \in \mathbb{Z})$ used in Section 5.2 to define dimensions is contained in $a_{t}^{-1} N(\mathfrak{o}) a_{t}$ for sufficiently large $t$, we see that $f^{-1} Y$ is ind-admissible and that

$$
\operatorname{dim} f^{-1} Y-\operatorname{dim} Y=d,
$$

as desired.

The last point is the surjectivity of $f$. We already know by Claim 1 that $f\left(N_{0}\right)$ contains $a N_{0} a^{-1}$. Therefore $f\left(a_{t}^{-1} N_{0} a_{t}\right)$ contains $a_{t}^{-1} a N_{0} a^{-1} a_{t}$ for all $t \in \mathbb{Z}$. Since the union of the subgroups $a_{t}^{-1} a N_{0} a^{-1} a_{t}(t \in \mathbb{Z})$ is all of $N(L)$, we see that $f$ is indeed surjective.

\subsection{Dimensions of intersections of $N(L)$ - and $K$-orbits on $X^{G}$}

As before we put $x_{\lambda}=\varepsilon^{\lambda} x_{0}$. Let $\mu$ be a $G$-dominant coweight.

For $m \in M(L)$ we are interested in the intersection

$$
N(L) m x_{0} \cap K x_{\mu}
$$

$4^{\text {e }}$ SÉRIE - TOME $39-2006-\mathrm{N}^{\circ} 3$ 
of the $N(L)$-orbit of $m x_{0}$ and the $K$-orbit of $x_{\mu}$ in the affine Grassmannian $X^{G}$. Let $\mu_{M}$ be the unique $M$-dominant coweight such that $m \in K_{M} \varepsilon^{\mu_{M}} K_{M}$. Then there exists $k_{M} \in K_{M}$ such that $m x_{0}=k_{M} x_{\mu_{M}}$, and left multiplication by $k_{M}$ defines an isomorphism

$$
N(L) x_{\mu_{M}} \cap K x_{\mu} \simeq N(L) m x_{0} \cap K x_{\mu} .
$$

For a given $G$-dominant coweight $\mu$ we write $S_{M}(\mu)$ for the set of $M$-dominant coweights $\mu_{M}$ for which the intersection $N(L) x_{\mu_{M}} \cap K x_{\mu}$ is non-empty. For $\nu \in \Lambda_{M}$ we then put

$$
S_{M}(\mu, \nu):=\left\{\mu_{M} \in S_{M}(\mu): p_{M}\left(\mu_{M}\right)=\nu\right\} .
$$

The next lemma will help us understand the finite sets $S_{M}(\mu)$ and $S_{M}(\mu, \nu)$. Define a finite subset $\Sigma(\mu)$ of $X_{*}(A)$ as follows: $\mu^{\prime} \in \Sigma(\mu)$ if and only if $\mu_{\text {dom }}^{\prime} \leqslant \mu$, where, as before, $\mu_{\text {dom }}^{\prime}$ denotes the unique $G$-dominant element in the $W$-orbit of $\mu^{\prime}$. Then denote by $\Sigma(\mu)_{M \text {-dom }}$ the set of $M$-dominant elements in $\Sigma(\mu)$. Finally, denote by $\Sigma(\mu)_{M \text {-max }}$ the set of all elements in $\Sigma(\mu)_{M \text {-dom }}$ that are maximal in $\Sigma(\mu)_{M \text {-dom }}$ with respect to the partial order $\underset{M}{\leqslant}$ on $X_{*}(A)$.

\section{LEMmA 5.4.1. - For any $G$-dominant coweight $\mu$ there are inclusions}

$$
\Sigma(\mu)_{M-\max } \subset S_{M}(\mu) \subset \Sigma(\mu)_{M-\text { dom }} .
$$

Moreover, all three sets $\Sigma(\mu)_{M-\max }, S_{M}(\mu), \Sigma(\mu)_{M \text {-dom }}$ have the same image under the map $p_{M}: X_{*}(A) \rightarrow \Lambda_{M}$. In particular $S_{M}(\mu, \nu)$ is non-empty if and only if $\nu$ lies in the image of $\Sigma(\mu)_{M-\text { dom }}$ under $p_{M}$.

Proof. - First recall [21] (see also [27,9]) that a coweight $\mu^{\prime}$ lies in $\Sigma(\mu)$ if and only if $U(L) \varepsilon^{\mu^{\prime}}$ meets $K \varepsilon^{\mu} K$. By definition $\mu^{\prime} \in S_{M}(\mu)$ if and only if $\mu^{\prime}$ is $M$-dominant and $N(L) \varepsilon^{\mu^{\prime}}$ meets $K \varepsilon^{\mu} K$. Since $N \subset U$, it is clear that $S_{M}(\mu) \subset \Sigma(\mu)_{M \text {-dom }}$.

Now let $\mu^{\prime} \in \Sigma(\mu)_{M \text {-max }}$. Thus there exists $u \in U(L)$ such that $u \varepsilon^{\mu^{\prime}} \in K \varepsilon^{\mu} K$. Write $u=n u_{M}$ with $n \in N(L), u_{M} \in U_{M}(L)$. Then $N(L) u_{M} \varepsilon^{\mu^{\prime}}$ meets $K \varepsilon^{\mu} K$, so there exists $\mu_{M} \in S_{M}(\mu)$ such that $u_{M} \varepsilon^{\mu^{\prime}} \in K_{M} \varepsilon^{\mu_{M}} K_{M}$. Now applying the fact recalled at the beginning of the proof to $M$ rather than $G$, we see that $\mu^{\prime} \underset{M}{\leqslant} \mu_{M}$, and of course $\mu_{M}$ lies in $\Sigma(\mu)_{M \text {-dom, }}$ since we have already proved that $S_{M}(\mu) \subset \Sigma(\mu)_{M \text {-dom }}$. By maximality of $\mu^{\prime}$ we then conclude that $\mu^{\prime}=\mu_{M}$, proving that $\mu^{\prime} \in S_{M}(\mu)$, as desired.

For the second statement of the lemma it is enough to show that $\Sigma(\mu)_{M \text {-max }}$ and $\Sigma(\mu)_{M \text {-dom }}$ have the same image under the map $p_{M}: X_{*}(A) \rightarrow \Lambda_{M}$. So suppose that $p_{M}(\mu)=\nu$ for some

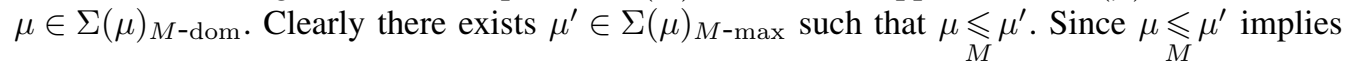
$p_{M}\left(\mu^{\prime}\right)=p_{M}(\mu)$, we are done.

The last statement of the lemma follows from the second statement.

For $G$-dominant $\mu$ and $\mu_{M} \in S_{M}(\mu)$ we denote by $d\left(\mu, \mu_{M}\right)$ the dimension of the intersection $N(L) x_{\mu_{M}} \cap K x_{\mu}$. From (5.4.2) we see that

$$
\operatorname{dim} N(L) m x_{0} \cap K x_{\mu}=d\left(\mu, \mu_{M}\right)
$$

for all $m \in K_{M} \varepsilon^{\mu_{M}} K_{M}$.

Our next task is to give an estimate for the numbers $d\left(\mu, \mu_{M}\right)$. At this point we need to introduce some new notation. Recall that we have fixed a Borel $B=A U$ in $G$, and this gives rise to a based root system. Let $G^{\vee} \supset B^{\vee} \supset A^{\vee}$ denote the complex reductive group together with a Borel and a maximal torus, whose root system is dual to that determined by $G \supset B \supset A$. 
The Levi subgroup $M$ is determined by a subset $\Delta_{M}$ of the simple $B$-positive roots for $G$. We let $M^{\vee}$ denote the Levi subgroup of $G^{\vee}$ determined by the set of simple $B^{\vee}$-positive roots $\left\{\alpha^{\vee} \mid \alpha \in \Delta_{M}\right\}$. Then $M^{\vee}$ is a dual group for $M$. Given a $G$-dominant coweight $\mu \in X_{*}(A)$, we will simultaneously think of it also as a weight $\mu \in X^{*}\left(A^{\vee}\right)$. Let $\chi_{\mu}^{G}$ denote the character of the unique irreducible $G^{\vee}$-module with highest weight $\mu$. For an $M$-dominant coweight $\mu_{M} \in X_{*}(A)$, the symbol $\chi_{\mu_{M}}^{M}$ has the analogous meaning.

Proposition 5.4.2. - Let $\mu$ be a $G$-dominant coweight. Then for all $\mu_{M} \in S_{M}(\mu)$ there is an inequality

$$
d\left(\mu, \mu_{M}\right) \leqslant\left\langle\rho, \mu+\mu_{M}\right\rangle-2\left\langle\rho_{M}, \mu_{M}\right\rangle,
$$

and equality holds if and only if $\chi_{\mu_{M}}^{M}$ occurs in the restriction of $\chi_{\mu}^{G}$ to $M^{\vee}$. Moreover, the number of irreducible components of the intersection $N(L) x_{\mu_{M}} \cap K x_{\mu}$ having dimension $\left\langle\rho, \mu+\mu_{M}\right\rangle-2\left\langle\rho_{M}, \mu_{M}\right\rangle$ is the multiplicity $a_{\mu_{M} \mu}$ with which $\chi_{\mu_{M}}^{M}$ occurs in $\chi_{\mu}^{G}$.

Proof. - To simplify notation, during this proof we temporarily use the symbol $\lambda$ instead of $\mu_{M}$ to denote an $M$-dominant coweight.

Recall that $F=k((\varepsilon))$ where $k$ denotes the finite field with $q$ elements. We will use the symbols $G, M, B, A$ etc. to denote the groups of $F$-points $G(F), M(F), B(F), A(F)$ etc., and furthermore here we write $K$ (respectively $K_{M}$ ) for the compact open subgroup $G(k[[\varepsilon]])$ (respectively $M(k[[\varepsilon]])$ of $G(F)$ (respectively $M(F)$ ). The intersection $N(F) x_{\lambda} \cap K x_{\mu}$ is the set of $k$-points of the variety $N(L) x_{\lambda} \cap G(\mathfrak{o}) x_{\mu}$, and an estimate for the dimension of the latter will follow from a suitable estimate for the growth of the number of points

$$
n(\lambda, \mu)(q)=\#\left(N(F) x_{\lambda} \cap K x_{\mu}\right)
$$

as a function of $q$. In fact we will show that $n(\lambda, \mu)(q)$ is a polynomial in $q$ with degree bounded above by $\langle\rho, \mu+\lambda\rangle-2\left\langle\rho_{M}, \lambda\right\rangle$. This is enough to prove the upper bound on the dimension of $N(L) x_{\lambda} \cap G(\mathfrak{o}) x_{\mu}$.

To prove this we will calculate $n(\lambda, \mu)(q)$ in terms of values of constant terms of standard spherical functions, and we will estimate those values by manipulating the Kato-Lusztig formula $[18,11]$. An exposition of this key ingredient, as well as the Satake isomorphism used below, can be found in [10].

Let $H_{K}(G)=C_{c}(K \backslash G / K)$ and $H_{K}(M)=C_{c}\left(K_{M} \backslash M / K_{M}\right)$ denote the spherical Hecke algebras of $G$ and $M$ respectively. Convolution is defined using the Haar measures giving $K$ respectively $K_{M}$ volume 1 . For a parabolic subgroup $P=M N$ of $G$, the constant term homomorphism $c_{M}^{G}: H_{K}(G) \rightarrow H_{K}(M)$ is defined by the formula

$$
c_{M}^{G}(f)(m)=\delta_{P}(m)^{-1 / 2} \int_{N} f(n m) \mathrm{d} n .
$$

Here $\delta_{P}(m):=|\operatorname{det}(\operatorname{Ad}(m) ; \operatorname{Lie}(N))|$, and the Haar measure on $N$ is such that $N \cap K$ has volume 1 . We define in a similar way $\delta_{B}, \delta_{B_{M}}, c_{A}^{G}$, and $c_{A}^{M}$. Recall that $U=U_{M} N$, and so

$$
\delta_{B}(t)=\delta_{P}(t) \delta_{B_{M}}(t)
$$

for $t \in A$, and

$$
c_{A}^{G}(f)(t)=\left(c_{A}^{M} \circ c_{M}^{G}\right)(f)(t)
$$

$4^{\text {e }}$ SÉRIE - TOME $39-2006-\mathrm{N}^{\circ} 3$ 
Also, $c_{A}^{G}$ (respectively $c_{A}^{M}$ ) is the Satake isomorphism $S^{G}$ for $G$ (respectively $S^{M}$ for $M$ ). Thus, the following diagram commutes:

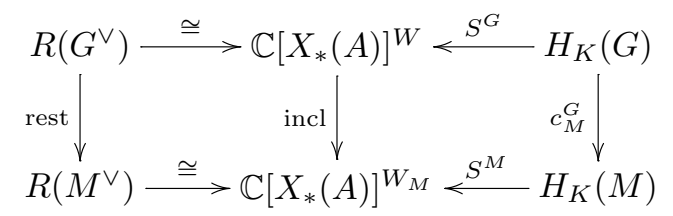

where $R\left(G^{\vee}\right)$ denotes the representation ring for $G^{\vee}$.

For a $G$-dominant coweight $\mu$, let $f_{\mu}^{G}=\operatorname{char}(K \mu K)$, and let $f_{\lambda}^{M}$ have the analogous meaning. For any $M$-dominant coweight $\lambda$ of $M$, we define numbers $a_{\lambda \mu}$ and $b_{\lambda \mu}(q)$ by the equalities

$$
\begin{aligned}
\operatorname{rest}\left(\chi_{\mu}^{G}\right) & =\sum_{\lambda} a_{\lambda \mu} \chi_{\lambda}^{M}, \\
c_{M}^{G}\left(f_{\mu}^{G}\right) & =\sum_{\lambda} b_{\lambda \mu}(q) f_{\lambda}^{M} .
\end{aligned}
$$

LEMMA 5.4.3. - Assume that $\lambda \in S_{M}(\mu)$.

(1) We have $b_{\lambda \mu}(q) \in \mathbb{Z}\left[q^{1 / 2}, q^{-1 / 2}\right]$, and $q^{-\langle\rho, \mu\rangle+\left\langle\rho_{M}, \lambda\right\rangle} b_{\lambda \mu}(q) \in \mathbb{Z}\left[q^{-1}\right]$.

(2) There is an equality

$$
b_{\lambda \mu}(q)=a_{\lambda \mu} q^{\langle\rho, \mu\rangle-\left\langle\rho_{M}, \lambda\right\rangle}+\left\{\text { terms with lower } q^{1 / 2} \text {-degree }\right\} .
$$

Proof. - Recall the Kato-Lusztig formula

$$
\chi_{\mu}^{G}=\sum_{\mu^{\prime} \leqslant \mu} q^{-\langle\rho, \mu\rangle} P_{w_{\mu^{\prime}}, w_{\mu}}(q) S^{G}\left(f_{\mu^{\prime}}^{G}\right),
$$

where $\mu^{\prime}$ ranges over $G$-dominant elements in $\Sigma(\mu), t_{\mu^{\prime}} \in \widetilde{W}$ denotes the corresponding translation element, and $w_{\mu^{\prime}}$ denotes the longest element in the double coset $W t_{\mu^{\prime}} W$. Of course $P_{w_{\mu^{\prime}}, w_{\mu}}(q)$ is the Kazhdan-Lusztig polynomial, and hence its $q$-degree is strictly bounded above by $\left\langle\rho, \mu-\mu^{\prime}\right\rangle$ if $\mu \neq \mu^{\prime}$. Also, $P_{w_{\mu}, w_{\mu}}(q)=1$.

The Kato-Lusztig formula for the group $M$ can be written

$$
\chi_{\lambda^{\prime}}^{M}=\sum_{\lambda} p_{\lambda \lambda^{\prime}}^{M}\left(q^{-1}\right) \frac{S^{M}\left(f_{\lambda}^{M}\right)}{q^{\left\langle\rho_{M}, \lambda\right\rangle}}
$$

for some polynomials $p_{\lambda, \lambda^{\prime}}^{M} \in \mathbb{Z}\left[q^{-1}\right]$ which vanish unless $\lambda \underset{M}{\leqslant} \lambda^{\prime}$. This expresses the relation between two bases of $R\left(M^{\vee}\right)$ given by an "upper triangular" invertible matrix. Inverting the corresponding relation for the group $G$ gives the formula

$$
\frac{S^{G}\left(f_{\mu}^{G}\right)}{q^{\langle\rho, \mu\rangle}}=\sum_{\mu^{\prime}} q_{\mu^{\prime} \mu}^{G}\left(q^{-1}\right) \chi_{\mu^{\prime}}^{G}
$$

for some $q^{G} \in \mathbb{Z}\left[q^{-1}\right]$. We find that

$$
\frac{S\left(f_{\mu}^{G}\right)}{q^{\langle\rho, \mu\rangle}}=\sum_{\mu^{\prime}} q_{\mu^{\prime} \mu}^{G} \chi_{\mu^{\prime}}^{G}=\sum_{\mu^{\prime}} \sum_{\lambda^{\prime}} a_{\lambda^{\prime} \mu^{\prime}} q_{\mu^{\prime} \mu}^{G} \chi_{\lambda^{\prime}}^{M}=\sum_{\mu^{\prime}, \lambda^{\prime}, \lambda} a_{\lambda^{\prime} \mu^{\prime}} q_{\mu^{\prime} \mu}^{G} p_{\lambda \lambda^{\prime}}^{M} \frac{S\left(f_{\lambda}^{M}\right)}{q^{\left\langle\rho_{M}, \lambda\right\rangle}} .
$$


We also have the relation

$$
\frac{S\left(f_{\mu}^{G}\right)}{q^{\langle\rho, \mu\rangle}}=\sum_{\lambda} \frac{b_{\lambda \mu}(q)}{q^{\langle\rho, \mu\rangle-\left\langle\rho_{M}, \lambda\right\rangle}} \frac{S\left(f_{\lambda}^{M}\right)}{q^{\left\langle\rho_{M}, \lambda\right\rangle}} .
$$

Thus we see that for each $\lambda \in S_{M}(\mu)$,

$$
\frac{b_{\lambda \mu}(q)}{q^{\langle\rho, \mu\rangle-\left\langle\rho_{M}, \lambda\right\rangle}}=\sum_{\mu^{\prime}, \lambda^{\prime}} a_{\lambda^{\prime} \mu^{\prime}} q_{\mu^{\prime} \mu}^{G} p_{\lambda \lambda^{\prime}}^{M}
$$

Part (1) follows from this equality, as does (2) once one notes that $q_{\mu^{\prime}, \mu}^{G}$ and $p_{\lambda, \lambda^{\prime}}^{M}$ as polynomials in $\mathbb{Z}\left[q^{-1}\right]$ have non-zero constant terms if and only if $\mu^{\prime}=\mu$ and $\lambda=\lambda^{\prime}$ respectively.

Now we continue with the proof of the proposition. Using the integration formula from the Iwasawa decomposition $G=K N M(y=k n m)$, we see that the number of points

$$
n(\lambda, \mu)(q)=\#\left(N \varepsilon^{\lambda} K / K \cap K \varepsilon^{\mu} K / K\right)
$$

is given by

$$
\begin{aligned}
n(\lambda, \mu)(q) & =\int_{G} 1_{N K}\left(\varepsilon^{-\lambda} y^{-1}\right) 1_{K \varepsilon^{-\mu} K}(y) \mathrm{d} y \\
& =\int_{M} \int_{N} \int_{K} 1_{N K}\left(\varepsilon^{-\lambda} m^{-1} n^{-1} k^{-1}\right) 1_{K \varepsilon^{-\mu} K}(k n m) \mathrm{d} k \mathrm{~d} n \mathrm{~d} m \\
& =\int_{M} \int_{N} 1_{N K}\left(m^{-1}\right) 1_{K \varepsilon^{-\mu} K}\left(n m \varepsilon^{-\lambda}\right) \mathrm{d} n \mathrm{~d} m \\
& =\int_{N} 1_{K \varepsilon^{-\mu} K}\left(n \varepsilon^{-\lambda}\right) \mathrm{d} n \\
& =\delta_{P}\left(\varepsilon^{-\lambda}\right)^{1 / 2} c_{M}^{G}\left(1_{K \varepsilon^{-w_{0}^{G} \mu} K}\right)\left(\varepsilon^{-\lambda}\right) \\
& =q^{\left\langle\rho_{N}, \lambda\right\rangle} c_{M}^{G}\left(1_{K \varepsilon^{-w_{0}^{G}} K_{K}}\right)\left(\varepsilon^{-w_{0}^{M} \lambda}\right) .
\end{aligned}
$$

Here $\rho_{N}$ is the half-sum of the positive roots occurring in $\operatorname{Lie}(N)$, and $w_{0}^{G}$ (respectively $w_{0}^{M}$ ) is the longest element in the Weyl group $W$ (respectively $W_{M}$ ).

Using Lemma 5.4.3, we see that

$$
\begin{aligned}
\operatorname{dim}\left(N(L) x_{\lambda} \cap G(\mathfrak{o}) x_{\mu}\right) & \leqslant\left\langle\rho_{N}, \lambda\right\rangle+\left\langle\rho,-w_{0}^{G} \mu\right\rangle-\left\langle\rho_{M},-w_{0}^{M} \lambda\right\rangle \\
& =\left\langle\rho-\rho_{M}, \lambda\right\rangle+\langle\rho, \mu\rangle-\left\langle\rho_{M}, \lambda\right\rangle \\
& =\langle\rho, \lambda+\mu\rangle-2\left\langle\rho_{M}, \lambda\right\rangle,
\end{aligned}
$$

and equality holds if and only if $\chi_{\lambda}^{M}$ is contained in $\chi_{\mu}^{G}$. Moreover, in that case, the number of irreducible components of the intersection having top dimension is the multiplicity $a_{\lambda \mu}$.

COROLLARY 5.4.4. - Let $\mu$ be a $G$-dominant coweight. Then for all $\mu_{M} \in S_{M}(\mu)$ there is an inequality

$$
d\left(\mu, \mu_{M}\right) \leqslant\left\langle\rho, \mu+\mu_{M}\right\rangle-2\left\langle\rho_{M}, \mu_{M}\right\rangle,
$$

and when $\mu_{M} \in \Sigma(\mu)_{M-\max }$, this inequality is an equality.

$4^{\text {e }}$ SÉRIE - TOME $39-2006-\mathrm{N}^{\circ} 3$ 
Proof. - If $\mu_{M} \in \Sigma(\mu)_{M-\max }$, then it is easy to see that $\chi_{\mu_{M}}^{M}$ appears in the restriction of $\chi_{\mu}^{G}$ to $M^{\vee}$.

\subsection{Remark}

Taking $M=A$ in Proposition 5.4.2, we recover the dimension formula of Mirković-Vilonen, Proposition 2.14.1.

\subsection{Reduction step}

Let $b \in M(L)$ and assume that $b$ is basic in $M(L)$. The map $\eta_{M}: M(L) \rightarrow \Lambda_{M}$ is constant on $\sigma$-conjugacy classes and induces a bijection between the set of basic $\sigma$-conjugacy classes in $M(L)$ and the group $\Lambda_{M}$. Put $\nu:=\eta_{M}(b) \in \Lambda_{M}$. We denote by $\bar{\nu} \in \mathfrak{a}_{M}$ the image of $\nu$ under the canonical homomorphism $\Lambda_{M} \rightarrow \mathfrak{a}_{M}$ (see (5.1.1)); since $b$ is basic, $\bar{\nu}$ is the Newton point of $b$.

For any $G$-dominant coweight $\mu$ we have the affine Deligne-Lusztig variety

$$
X_{\mu}^{G}(b)=\left\{x \in G(L) / K: x^{-1} b \sigma(x) \in K \varepsilon^{\mu} K\right\},
$$

which may or may not be non-empty. Moreover, for any $M$-dominant coweight $\mu_{M}$ we have the affine Deligne-Lusztig variety

$$
X_{\mu_{M}}^{M}(b)=\left\{x \in M(L) / K_{M}: x^{-1} b \sigma(x) \in K_{M} \varepsilon^{\mu_{M}} K_{M}\right\} .
$$

Since $b$ is basic in $M(L)$ the affine Deligne-Lusztig variety $X_{\mu_{M}}^{M}(b)$ is non-empty if and only if $p_{M}\left(\mu_{M}\right)=\nu$ (see [16, Prop. 4.1], and note that its proof works for any split group, or see [15, Thm. 4.3]).

Our goal is to express $\operatorname{dim} X_{\mu}^{G}(b)$ in terms of the numbers $\operatorname{dim} X_{\mu_{M}}^{M}(b)$ and $d\left(\mu, \mu_{M}\right)$ for the various coweights $\mu_{M} \in S_{M}(\mu, \nu)$, and then to use this expression to reduce Rapoport's dimension conjecture to the basic case.

We will use the following map $\alpha: X^{G} \rightarrow X^{M}$. By the Iwasawa decomposition we have $G(L)=P(L) K$. Therefore there is an obvious bijection

$$
P(L) / K_{P} \simeq G(L) / K,
$$

where $K_{P}:=P(\mathfrak{o})$. The canonical surjective homomorphism $P(L) \rightarrow M(L)$ induces a surjection

$$
P(L) / K_{P} \rightarrow M(L) / K_{M} .
$$

The map $\alpha$ is defined as the composition

$$
\alpha: X^{G}=G(L) / K \simeq P(L) / K_{P} \rightarrow M(L) / K_{M}=X^{M} .
$$

We hasten to warn the reader that $\alpha$ is not a morphism of ind-schemes. However, for any connected component $Y$ of $X^{M}$, the inverse image $\alpha^{-1} Y$ is a locally closed subset of $X^{G}$, and the map $\alpha^{-1} Y \rightarrow Y$ obtained by restriction from $\alpha$ is a morphism of ind-schemes. For $m \in M(L)$ we can identify the fiber $\alpha^{-1}\left(m x_{0}\right)$ with $N(L) / N(\mathfrak{o})$ via

$$
N(L) / N(\mathfrak{o}) \ni n \mapsto m n x_{0} \in \alpha^{-1}\left(m x_{0}\right) .
$$

PROPOSITION 5.6.1. - Assume that the $\sigma$-conjugacy class of $b$ in $M(L)$ is basic, and let $\nu$ be the element $\nu=\eta_{M}(b) \in \Lambda_{M}$. 
(1) The space $X_{\mu}^{G}(b)$ is non-empty if and only if $\nu$ lies in the image of the set $\Sigma(\mu)_{M \text {-dom. }}$.

(2) The image of $X_{\mu}^{G}(b)$ under $\alpha$ is contained in

$$
\bigcup_{\mu_{M} \in S_{M}(\mu, \nu)} X_{\mu_{M}}^{M}(b)
$$

Denote by $\beta$ the map

$$
\beta: X_{\mu}^{G}(b) \rightarrow \bigcup_{\mu_{M} \in S_{M}(\mu, \nu)} X_{\mu_{M}}^{M}(b)
$$

obtained by restriction from $\alpha$.

(3) For $\mu_{M} \in S_{M}(\mu, \nu)$ and $x \in X_{\mu_{M}}^{M}(b)$ the fiber $\beta^{-1}(x)$ is non-empty and

$$
\operatorname{dim} \beta^{-1}(x)=d\left(\mu, \mu_{M}\right)+\left\langle\rho, \bar{\nu}-\bar{\nu}_{\operatorname{dom}}\right\rangle-\left\langle 2 \rho_{N}, \bar{\nu}\right\rangle .
$$

In particular $\beta$ is surjective.

(4) For all $\mu_{M} \in S_{M}(\mu, \nu)$ and all $\lambda \in \Lambda_{M}$ the set $\beta^{-1}\left(X_{\mu_{M}}^{M}(b) \cap \eta_{M}^{-1}(\lambda)\right)$ is locally closed in $X_{\mu}^{G}(b)$, and

$$
\operatorname{dim} \beta^{-1}\left(X_{\mu_{M}}^{M}(b) \cap \eta_{M}^{-1}(\lambda)\right)=\operatorname{dim} X_{\mu_{M}}^{M}(b)+d\left(\mu, \mu_{M}\right)+\left\langle\rho, \bar{\nu}-\bar{\nu}_{\operatorname{dom}}\right\rangle-\left\langle 2 \rho_{N}, \bar{\nu}\right\rangle .
$$

(5) If $X_{\mu}^{G}(b)$ is non-empty, its dimension is given by

$$
\sup \left\{\operatorname{dim} X_{\mu_{M}}^{M}(b)+d\left(\mu, \mu_{M}\right): \mu_{M} \in S_{M}(\mu, \nu)\right\}+\left\langle\rho, \bar{\nu}-\bar{\nu}_{\mathrm{dom}}\right\rangle-\left\langle 2 \rho_{N}, \bar{\nu}\right\rangle .
$$

Proof. - We begin by proving (2). Let $x \in X_{\mu}^{G}(b)$. Write $x=m n x_{0}$ with $m \in M(L)$, $n \in N(L)$. Then

$$
n^{-1} m^{-1} b \sigma(m) \sigma(n) \in K \varepsilon^{\mu} K,
$$

from which it follows that $m^{-1} b \sigma(m)$ lies in $K_{M} \varepsilon^{\mu_{M}} K_{M}$ for a unique $\mu_{M} \in S_{M}(\mu)$. Thus $\alpha(x)=m x_{0} \in X_{\mu_{M}}^{M}(b)$, showing that $X_{\mu_{M}}^{M}(b)$ is non-empty and hence that $\mu_{M} \in S_{M}(\mu, \nu)$. This proves (2).

Next we prove (3). Let $\mu_{M} \in S_{M}(\mu, \nu)$ and $x \in X_{\mu_{M}}^{M}(b)$. Choose $m \in M(L)$ such that $x=m x_{0}$. Then $b^{\prime}:=m^{-1} b \sigma(m) \in K_{M} \varepsilon^{\mu_{M}} K_{M}$ and

$$
\beta^{-1}(x)=f_{b^{\prime}}^{-1}\left(K \varepsilon^{\mu} K b^{\prime-1} \cap N(L)\right) / N(\mathfrak{o}),
$$

where $f_{b^{\prime}}$ is the morphism $N(L) \rightarrow N(L)$ defined by

$$
f_{b^{\prime}}(n)=n^{-1} b^{\prime} \sigma(n) b^{\prime-1} .
$$

Since $f_{b^{\prime}}$ is surjective, the fiber $\beta^{-1}(x)$ is non-empty.

Since $b^{\prime}$ is $\sigma$-conjugate to $b$ in $M(L)$, its Newton point is also $\bar{\nu}$. Therefore Proposition 5.3.1 tells us that

$$
\operatorname{dim} \beta^{-1}(x)=\operatorname{dim}\left(K \varepsilon^{\mu} K b^{\prime-1} \cap N(L)\right)+\left\langle\rho, \bar{\nu}-\bar{\nu}_{\text {dom }}\right\rangle .
$$

Furthermore

$$
N(L) b^{\prime} x_{0} \cap K x_{\mu}=\left(K \varepsilon^{\mu} K b^{\prime-1} \cap N(L)\right) / b^{\prime} N(\mathfrak{o}) b^{\prime-1},
$$

$4^{\text {e }}$ SÉRIE - TOME $39-2006-\mathrm{N}^{\circ} 3$ 
from which it follows that

$$
\operatorname{dim}\left(K \varepsilon^{\mu} K b^{\prime-1} \cap N(L)\right)=d\left(\mu, \mu_{M}\right)+\operatorname{dim}\left(b^{\prime} N(\mathfrak{o}) b^{-1}\right),
$$

because $b^{\prime} \in K_{M} \varepsilon^{\mu_{M}} K_{M}$. Since $\operatorname{dim} b^{\prime} N(\mathfrak{o}) b^{-1}$ is obviously equal to $-\left\langle 2 \rho_{N}, \bar{\nu}\right\rangle$, we conclude that

$$
\operatorname{dim} \beta^{-1}(x)=d\left(\mu, \mu_{M}\right)+\left\langle\rho, \bar{\nu}-\bar{\nu}_{\mathrm{dom}}\right\rangle-\left\langle 2 \rho_{N}, \bar{\nu}\right\rangle .
$$

This proves (3), and (1) follows from (2), (3), Lemma 5.4.1 and the fact that $X_{\mu_{M}}^{M}$ (b) is nonempty for all $\mu_{M} \in S_{M}(\mu, \nu)$.

Since $\operatorname{dim} \beta^{-1}(x)$ is independent of $x \in X_{\mu_{M}}^{M}(b)$, part (4) follows from part (3) and the fact (noted already in the introduction) that

$$
\operatorname{dim}\left(X_{\mu_{M}}^{M}(b) \cap \eta_{M}^{-1}(\lambda)\right)=\operatorname{dim} X_{\mu_{M}}^{M}(b)
$$

for all $\lambda \in \Lambda_{M}$.

Finally we prove (5). Assume that $X_{\mu}^{G}(b)$ is non-empty, so that $S_{M}(\mu, \nu)$ is non-empty as well. Then $X_{\mu}^{G}(b)$ is set-theoretically the union of the locally closed pieces

$$
\beta^{-1}\left(X_{\mu_{M}}^{M}(b) \cap \eta_{M}^{-1}(\lambda)\right)
$$

where $\mu_{M}$ ranges over the finite set $S_{M}(\mu, \nu)$ and $\lambda$ ranges through $\Lambda_{M}$. The space $X_{\mu}^{G}(b)$ is preserved by the action of $A_{M}(F)$, and the action of $a \in A_{M}(F)$ on $X_{\mu}^{G}(b)$ sends $\beta^{-1}\left(X_{\mu_{M}}^{M}(b) \cap \eta_{M}^{-1}(\lambda)\right)$ to $\beta^{-1}\left(X_{\mu_{M}}^{M}(b) \cap \eta_{M}^{-1}\left(\lambda+\lambda_{a}\right)\right)$, where $\lambda_{a}:=\eta_{M}(a)$. Since $\eta_{M}\left(A_{M}(F)\right)$ has finite index in $\Lambda_{M}$, the action of $A_{M}(F)$ has finitely many orbits on the set of pieces in our decomposition. Reasoning as in Section 2.5 , we conclude that $\operatorname{dim} X_{\mu}^{G}(b)$ is equal to the supremum of the dimensions of these pieces, and so we obtain (5) from (4).

\subsection{Remark}

Part (1) of the proposition we just proved is not new; it was proved in [16]. Moreover the strategy used to prove the proposition is simply a refinement of the method used to prove nonemptiness in [16].

\subsection{Reduction of Rapoport's dimension conjecture to the basic case}

We continue with $G$-dominant $\mu$ and a basic element $b \in M(L)$, as above. We again use $b$ to get $\nu \in \Lambda_{M}$ and the Newton point $\bar{\nu} \in \mathfrak{a}_{M}$.

We write $\operatorname{def}_{M}(b)$ for the defect of $b$, a non-negative integer no greater than the semisimple rank of $M$. By definition $\operatorname{def}_{M}(b)$ is simply the $F$-rank of $M$ minus the $F$-rank of the inner form $J$ of $M$ obtained by twisting $M$ by $b$. (The group $J(F)$ is the $\sigma$-centralizer of $b$ in $M(L)$.) Rapoport's conjecture (applied to $M$ and the basic element $b$ ) states that for all $M$-dominant $\mu_{M}$ with $p_{M}\left(\mu_{M}\right)=\nu$ the dimension of $X_{\mu_{M}}^{M}(b)$ is given by

$$
\operatorname{dim} X_{\mu_{M}}^{M}(b)=\left\langle\rho_{M}, \mu_{M}\right\rangle-\frac{1}{2} \operatorname{def}_{M}(b)
$$

Note that $\left\langle\rho_{M}, \mu_{M}\right\rangle$ and $\operatorname{def}_{M}(b) / 2$ lie in $\frac{1}{2} \mathbb{Z}$, but that their difference is always an integer.

Let us assume this formula is true and combine it with Proposition 5.6.1 in order to calculate $\operatorname{dim} X_{\mu}^{G}(b)$. Recall that $X_{\mu}^{G}(b)$ is non-empty if and only if $\nu$ lies in the image of $\Sigma(\mu)_{M \text {-dom. }}$. 
THEOREm 5.8.1. - Suppose that $X_{\mu}^{G}(b)$ is non-empty. Assume that (5.8.1) is true for all $\mu_{M} \in S_{M}(\mu, \nu)$. Then the dimension of $X_{\mu}^{G}(b)$ is given by

$$
\operatorname{dim} X_{\mu}^{G}(b)=\left\langle\rho, \mu-\bar{\nu}_{\mathrm{dom}}\right\rangle-\frac{1}{2} \operatorname{def}_{G}(b)
$$

Proof. - By Proposition 5.6.1 $\operatorname{dim} X_{\mu}^{G}(b)$ is the sum of

$$
\left\langle\rho, \bar{\nu}-\bar{\nu}_{\mathrm{dom}}\right\rangle-\left\langle 2 \rho_{N}, \bar{\nu}\right\rangle
$$

and the supremum of the numbers

$$
\operatorname{dim} X_{\mu_{M}}^{M}(b)+d\left(\mu, \mu_{M}\right)
$$

as $\mu_{M}$ ranges through $S_{M}(\mu, \nu)$.

Combining Rapoport's dimension conjecture (5.8.1) with Corollary 5.4.4, we see that the number (5.8.2) is bounded above by

$$
\left\langle\rho_{M}, \mu_{M}\right\rangle-\operatorname{def}_{M}(b) / 2+\left\langle\rho, \mu+\mu_{M}\right\rangle-2\left\langle\rho_{M}, \mu_{M}\right\rangle
$$

with equality whenever $\mu_{M} \in \Sigma(\mu)_{M \text {-max }}$. Using the obvious equality $\left\langle\rho_{N}, \mu_{M}\right\rangle=\left\langle\rho_{N}, \bar{\nu}\right\rangle$, the displayed expression above simplifies to

$$
\langle\rho, \mu\rangle+\left\langle\rho_{N}, \bar{\nu}\right\rangle-\operatorname{def}_{M}(b) / 2,
$$

a number which is independent of $\mu_{M}$. Since we have supposed that $X_{\mu}^{G}(b)$ is non-empty, $\nu$ lies

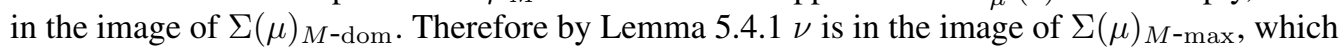
shows that the supremum of the numbers (5.8.2) is equal to the last displayed expression. Adding $\left\langle\rho, \bar{\nu}-\bar{\nu}_{\text {dom }}\right\rangle-\left\langle 2 \rho_{N}, \bar{\nu}\right\rangle$ to this, and using that $\left\langle\rho_{M}, \bar{\nu}\right\rangle=0$ since $\bar{\nu} \in \mathfrak{a}_{M}$, we find that

$$
\operatorname{dim} X_{\mu}^{G}(b)=\left\langle\rho, \mu-\bar{\nu}_{\text {dom }}\right\rangle-\operatorname{def}_{M}(b) / 2 .
$$

Now it remains only to observe that $\operatorname{def}_{M}(b)=\operatorname{def}_{G}(b)$. Indeed, the $F$-rank of a reductive group is the same as that of any of its Levi subgroups, so the $F$-ranks of $G$ and $M$ are the same. Moreover, the group $J$ attached to $(M, b)$ is a Levi subgroup of the group $J_{G}$ attached to $(G, b)$, so their $F$-ranks are the same.

\subsection{Reduction to the superbasic case}

Let $b \in G(L)$. As in the introduction $b$ determines a homomorphism $\nu_{b}: \mathbb{D} \rightarrow G$ over $L$. As in $[13,(4.4 .3)]$ we then have

$$
\operatorname{Int}(b) \circ \sigma\left(\nu_{b}\right)=\nu_{b} .
$$

Replacing $b$ by a $\sigma$-conjugate, we again assume that $\nu_{b}$ factors through $A$, which guarantees that $\sigma\left(\nu_{b}\right)=\nu_{b}$. Eq. (5.9.1) then says that $b \in M(L)$, where $M$ is the Levi subgroup of $G$ over $F$ obtained as the centralizer of $\nu_{b}$ in $G$. Since $\nu_{b}$ is central in $M$, the element $b$ is basic in $M(L)$. We have seen that if Rapoport's dimension conjecture is true for $(M, b)$, then it is true for $(G, b)$.

Thus it is enough to prove Rapoport's conjecture when $b$ is basic. Of course it may still happen that a basic element $b$ is contained in a proper Levi subgroup of $G$. For example this happens when $b=1$, as long as $G$ is not a torus. We say that an element $b \in G(L)$ is superbasic if no 
$\sigma$-conjugate of $b$ lies in a proper Levi subgroup of $G$ over $F$. Clearly superbasic elements are basic.

Suppose that $b \in G(L)$ is basic. Then the group $J$ discussed in the introduction is the inner form of $G$ obtained by twisting the action of $\sigma$ by $b$, so that

$$
J(F)=\left\{g \in G(L): g^{-1} b \sigma(g)=b\right\},
$$

or in other words $J(F)$ is the centralizer of $b \sigma$ in $G(L)$.

LEMMA 5.9.1. - The basic element $b$ is superbasic if and only if $J$ is anisotropic modulo the center $Z(G)$ of $G$.

Proof. - The group $J / Z(G)$ is not anisotropic if and only if there exists a non-central homomorphism $\mathbb{G}_{m} \rightarrow J$ which is defined over $F$. This happens if and only if there exist noncentral $\mu \in X_{*}(A)$ and an element $g \in G(L)$ such that $b \sigma$ and $\operatorname{Int}(g) \circ \mu$ commute. Now $b \sigma$ and $\operatorname{Int}(g) \circ \mu$ commute if and only if $g^{-1}(b \sigma) g$ and $\mu$ commute, and this happens if and only if $g^{-1} b \sigma(g)$ and $\mu$ commute (since $\sigma$ and $\mu$ commute), which is the same as saying that $g^{-1} b \sigma(g)$ lies in the Levi subgroup of $G$ obtained as the centralizer of $\mu$.

It follows from Theorem 5.8.1 that it is enough to prove Rapoport's conjecture when $b$ is superbasic. In the introduction we observed that the intersections of $X_{\mu}(b)$ with the various connected components of the affine Grassmannian are all isomorphic to each other. Let us denote by $X_{\mu}(b)_{\lambda}$ the intersection of $X_{\mu}(b)$ with the connected component of the affine Grassmannian indexed by $\lambda \in \Lambda_{G}$. It is easy to see that

$$
X_{\mu}(b)_{\lambda}=X_{\mu_{\mathrm{ad}}}\left(b_{\mathrm{ad}}\right)_{\lambda_{\mathrm{ad}}}
$$

where the subscript "ad" is being used to indicate the corresponding objects for the adjoint group $G / Z(G)$ of $G$. Thus it is enough to prove Rapoport's conjecture for superbasic elements in adjoint groups.

Any adjoint group is a direct product of simple groups. Therefore it is enough to prove Rapoport's conjecture for superbasic elements in simple (split) groups. From Lemma 5.9.1 we know that a basic element is superbasic if and only if the inner form $J$ of our simple group $G$ is anisotropic. This can happen only when $G$ is of type $A_{n}$, and then it is easy to see which elements are superbasic. Thus we obtain

PROPOSITION 5.9.2. - If Rapoport's conjecture is true for all basic elements $b$ in $G L_{n}(L)$ such that the valuation of $\operatorname{det}(b)$ is relatively prime to $n$, then Rapoport's conjecture is true in general.

Proof. - Observe that $G L_{n}(L) \rightarrow P G L_{n}(L)$ is surjective, and that a basic element $b \in$ $G L_{n}(L)$ is superbasic if and only if $\operatorname{val}(\operatorname{det} b)$ is relatively prime to $n$.

Shortly after this paper appeared as a preprint, Viehmann [34] proved Rapoport's conjecture for superbasic elements, and so Rapoport's conjecture is now proved in general.

\subsection{Relation of Rapoport's formula with Newton strata in Shimura varieties}

Suppose the data $(G, K, \mu)$ are the $p$-adic data coming from a PEL Shimura variety $S h$ with hyperspecial level structure at $p$. Then the variety $X_{\mu}(b)$ studied in this article has a $p$-adic variant $X_{\mu}(b)_{K}$ which relates to a certain moduli space $\mathcal{M}$ of $p$-divisible groups attached to $G, K, \mu$ and an element $b \in G\left(\widehat{\mathbb{Q}}_{p}^{u n}\right)$. There is a bijection $X_{\mu}(b)_{K}\left(\widehat{\mathbb{Q}}_{p}^{u n}\right)=\mathcal{M}_{\text {red }}\left(\overline{\mathbb{F}}_{p}\right)$. 
The element $b$ gives rise (under certain conditions) to a Newton stratum $\mathcal{S}(b) \subset S h \times \overline{\mathbb{F}}_{p}$.

Rapoport was inspired by Chai's conjecture in [3, Theorem 7.4], for the dimension of the Newton stratum $\mathcal{S}(b)$. Using [12], Chai's conjectural formula can be written in a manner which is strikingly similar to Rapoport's formula:

$$
\operatorname{dim} \mathcal{S}(b)=\left\langle\rho, \mu+\bar{\nu}_{b}\right\rangle-\frac{1}{2} \operatorname{def}_{G}(b) .
$$

Moreover, using Oort's [26] and Mantovan's [8,20] description of $\mathcal{S}(b)$ as the product (up to a finite morphism) of the Rapoport-Zink space $\mathcal{M}$ and the "central leaf" $\mathcal{C}(b)$ in $\mathcal{S}(b)$, we should have

$$
\operatorname{dim} \mathcal{C}(b)=\operatorname{dim} \mathcal{S}(b)-\operatorname{dim} X_{\mu}(b) .
$$

Thus Rapoport's formula and the above remarks imply the following remarkable formula for the dimension of the central leaf in terms of the Newton point:

$$
\operatorname{dim} \mathcal{C}(b)=\left\langle 2 \rho, \bar{\nu}_{b}\right\rangle
$$

(comp. with the formula of Chai-Oort in [26, Remark 2.8]). This formula is consistent with Table 8.1 in [26], which gives the dimensions of all central leaves in the Newton strata for the Siegel modular variety for $\operatorname{GSp}(8)$, with hyperspecial level structure at $p$.

\section{Affine Deligne-Lusztig varieties inside the affine flag manifold}

\subsection{Statement of the problem}

We continue with $G, A, W$ as before, but make a few changes in notation. We write $\mathcal{B}(A)$ for the set of Borel subgroups $B=A U$ containing $A$. We fix an alcove in the apartment $\mathfrak{a}:=X_{*}(A)_{\mathbb{R}}$ associated to $A$, and we denote by $I$ the corresponding Iwahori subgroup of $G(L)$. Note that $A(\mathfrak{o}) \subset I$. We write $\widetilde{W}$ for the affine Weyl group $W \ltimes X_{*}(A)=N_{G(L)}(A) / A(\mathfrak{o})$.

We will now let $X$ denote the affine flag manifold $G(L) / I$ and $\mathbf{a}_{1}$ its base point. For $x \in \widetilde{W}$ we put $\mathbf{a}_{x}:=x \mathbf{a}_{1} \in X$. Recall that

$$
X=\coprod_{x \in \widetilde{W}} I \mathbf{a}_{x}
$$

and that for any $B=A U \in \mathcal{B}(A)$

$$
X=\coprod_{x \in \widetilde{W}} U(L) \mathbf{a}_{x}
$$

Given $b \in G(L)$ and $x \in \widetilde{W}$, we get the affine Deligne-Lusztig variety

$$
X_{x}(b):=\left\{g \in G(L) / I: g^{-1} b \sigma(g) \in I x I\right\},
$$

a locally closed subset of $X$, possibly empty, whose dimension we are going to compute for all $b$ of the form $b=\varepsilon^{\nu}$ for some $\nu \in X_{*}(A)$. However the calculation will in fact only express $\operatorname{dim} X_{x}\left(\varepsilon^{\nu}\right)$ in terms of the (unknown) dimensions of intersections of $I$ - and $U(L)$-orbits in $X$. This is at least satisfactory for computer experiments, since the dimensions of these intersections can be determined by an algorithm involving only $\widetilde{W}$ and the set of affine roots.

$4^{\text {e }}$ SÉRIE - TOME $39-2006-\mathrm{N}^{\circ} 3$ 
Here is the algorithm (see [4] and [1, Prop. 2.3.12]). To simplify the description, let us assume that $G$ is semisimple. Consider the $I$-orbit of $\mathbf{a}_{x}$, which is an affine space $\mathbb{A}$ of dimension $\ell(x)$. Choose a reduced expression $x=s_{i_{1}} \cdots s_{i_{\ell(x)}}$. Equivalently, choose a minimal gallery $\mathcal{G}=\left(\mathbf{a}_{1}, \mathbf{a}_{s_{i_{1}}}, \ldots, \mathbf{a}_{x}\right)$ joining $\mathbf{a}_{1}$ to $\mathbf{a}_{x}$. (Here $\mathbf{a}_{1}$ is the alcove fixed by our Iwahori $I$, and the notions of length and reduced expression are defined using the simple reflections through the walls of $\mathbf{a}_{1}$.)

This choice of reduced expression defines an isomorphism of $\mathbb{A}$ with the product of a succession of affine lines, one for each wall between two alcoves of $\mathcal{G}$. In other words, $I \mathbf{a}_{x}$ consists of the end-alcoves of all galleries of the same type as $\mathcal{G}$. For each alcove in $I \mathbf{a}_{x}$ there is a unique gallery of the fixed type joining $\mathbf{a}_{1}$ to it, and associating to each alcove the last but one alcove in this gallery, we get a projection map pr: $\mathbb{A} \rightarrow I \mathbf{a}_{s_{i_{1}} \cdots s_{i_{\ell(x)-1}}} \cong \mathbb{A}^{\ell(x)-1}$ with fibres $\mathbb{A}^{1}$.

We will now decompose this affine space into finitely many pieces, each of which is contained in a single $U(L)$-orbit, by specifying which $U(L)$-orbit a point $y \in \mathbb{A}$ is in.

To the Borel $B=A U$ corresponds a Weyl chamber $C_{U}$, which is the unique one with the property that $A(\mathfrak{o}) U(L)$ is the union of the fixers in $G(L)$ of all "quartiers" of the form $x+C_{U}$, for $x \in X_{*}(A)_{\mathbb{R}}$ (see $\left.[1,4.1 .5,4.4 .4]\right)$. We can use any alcove in $C_{U}$ to retract $y$ into the standard apartment. The result depends on the choice of alcove, but for alcoves deep inside $C_{U}$, all retractions of $y$ are the same (cf. [1, 2.9.1]). It is a direct consequence of the above description of $C_{U}$ that the alcove $\rho_{U}(y)$ obtained as this common retraction of $y$ lies in the $U(L)$-orbit of $y$, telling us which $U(L)$-orbit $y$ belongs to.

Now for $y \in \mathbb{A}$, let $\tilde{y} \in I \mathbf{a}_{s_{i_{1}} \cdots s_{i_{\ell(x)-1}}}$ denote the last but one alcove in the unique gallery of the same type as $\mathcal{G}$ joining $\mathbf{a}_{1}$ to $y$. Let $\mathbf{a}_{U, \infty}$ be an alcove far out in the chamber $C_{U}$. By induction, we may assume that we know $\rho_{U}(\tilde{y})$. There are two possibilities for $\rho_{U}(y)$ : either it is equal to $\rho_{U}(\tilde{y})$, or it is the alcove $\mathbf{b}$ (in the standard apartment) adjacent to $\rho_{U}(\tilde{y})$ along the facet of type $i_{\ell(x)}$. Which of these two it is depends on $y$. However, if we now fix $\tilde{y}$ and consider all $y \in \operatorname{pr}^{-1}(\tilde{y})$ simultaneously, then we can give a precise description: If $\rho_{U}(\tilde{y})$ is closer to $\mathbf{a}_{U, \infty}$ than $\mathbf{b}$ is, then $\rho_{U}(y)=\mathbf{b}$ for all $y \in \operatorname{pr}^{-1}(\tilde{y})$. Otherwise, $\rho_{U}(y)=\mathbf{b}$ for a single point $y \in \operatorname{pr}^{-1}(\tilde{y})$, and $\rho_{U}(y)=\rho_{U}(\tilde{y})$ for all other points (see [1, Prop. 2.3.12 and Prop. 2.9.1]).

Keeping track of all these folding possibilities gives a decomposition of $\mathbb{A}$ into finitely many pieces, each of which is contained in a single $U(L)$-orbit and is a product of affine lines and affine lines minus a point. Taking the supremum of the dimensions of the pieces lying in a given $U(L)$-orbit, we obtain the dimension of the intersection of $\mathbb{A}$ with that $U(L)$-orbit.

\subsection{Intersections of $I$ - and $U(L)$-orbits}

Let $B=A U \in \mathcal{B}(A)$ and let $x, y \in \widetilde{W}$. Define a non-negative integer $d(x, y, B)$ by

$$
d(x, y, B):=\operatorname{dim} I \mathbf{a}_{x} \cap U(L) \mathbf{a}_{y} .
$$

(We set $d(x, y, B)=-\infty$ if this intersection is empty.) It is clear that

$$
I \mathbf{a}_{x} \cap U(L) \mathbf{a}_{y}=\left(I x I y^{-1} \cap U(L)\right) /\left(U(L) \cap y I y^{-1}\right),
$$

and hence that

$$
d(x, y, B)=\operatorname{dim} I x I y^{-1} \cap U(L)-\operatorname{dim} U(L) \cap y I y^{-1} .
$$

Here we are again using our dimension theory for $U(L)$, it being obvious that both $I x I y^{-1} \cap$ $U(L)$ and $U(L) \cap y I y^{-1}$ are admissible subsets of $U(L)$. 
In the special case $y=\varepsilon^{\nu}$ with $\nu \in X_{*}(A)$, we have

$$
U(L) \cap y I y^{-1}=\varepsilon^{\nu}(U(L) \cap I) \varepsilon^{-\nu}
$$

and hence

$$
\operatorname{dim}\left(U(L) \cap y I y^{-1}\right)=\operatorname{dim}(U(L) \cap I)-2\left\langle\rho_{B}, \nu\right\rangle,
$$

where $\rho_{B}$ is the half-sum of the roots of $A$ that are positive for $B$. Therefore we conclude that

$$
\operatorname{dim}\left(I x I \varepsilon^{-\nu} \cap U(L)\right)-\operatorname{dim}(U(L) \cap I)=d\left(x, \varepsilon^{\nu}, B\right)-2\left\langle\rho_{B}, \nu\right\rangle .
$$

\subsection{Computation of $\operatorname{dim} X_{x}\left(\varepsilon^{\nu}\right)$}

Pick $B=A U \in \mathcal{B}(A)$. As before we use the fact that $X_{x}\left(\varepsilon^{\nu}\right)$ is $A(F)$-stable to compute its dimension. The only difference is that $A(F)$ does not permute the $U(L)$-orbits $U(L) \mathbf{a}_{y}$ transitively. Nevertheless we have the only slightly more complicated formula

$$
\operatorname{dim} X_{x}\left(\varepsilon^{\nu}\right)=\sup _{w \in W} \operatorname{dim} X_{x}\left(\varepsilon^{\nu}\right) \cap U(L) \mathbf{a}_{w}
$$

Clearly left multiplication by $w^{-1}$ gives an isomorphism from

$$
X_{x}\left(\varepsilon^{\nu}\right) \cap U(L) \mathbf{a}_{w}
$$

to

$$
X_{x}\left(\varepsilon^{w^{-1} \nu}\right) \cap w^{-1} U(L) w \mathbf{a}_{1}
$$

and hence

$$
\operatorname{dim} X_{x}\left(\varepsilon^{\nu}\right)=\sup _{w \in W} \operatorname{dim} X_{x}\left(\varepsilon^{w \nu}\right) \cap w U w^{-1}(L) \mathbf{a}_{1} .
$$

So we need to understand $\operatorname{dim} X_{x}\left(\varepsilon^{\nu}\right) \cap U(L) \mathbf{a}_{1}$ for all $\nu \in X_{*}(A)$ and all $B=A U \in \mathcal{B}(A)$. Again writing $f_{\nu}$ for the map $u \mapsto u^{-1} \varepsilon^{\nu} \sigma(u) \varepsilon^{-\nu}$ from $U(L)$ to itself, we have

$$
X_{x}\left(\varepsilon^{\nu}\right) \cap U(L) \mathbf{a}_{1}=f_{\nu}^{-1}\left(I x I \varepsilon^{-\nu} \cap U(L)\right) / U(L) \cap I
$$

and hence

$$
\operatorname{dim} X_{x}\left(\varepsilon^{\nu}\right) \cap U(L) \mathbf{a}_{1}=\operatorname{dim} f_{\nu}^{-1}\left(I x I \varepsilon^{-\nu} \cap U(L)\right)-\operatorname{dim} U(L) \cap I
$$

Using Proposition 2.12.1, Lemma 2.13.1 and (6.2.4), we see that

$$
\operatorname{dim} f_{\nu}^{-1}\left(I x I \varepsilon^{-\nu} \cap U(L)\right)-\operatorname{dim} U(L) \cap I=d\left(x, \varepsilon^{\nu}, B\right)-\left\langle\rho_{B}, \nu+\nu_{B}\right\rangle,
$$

where $\nu_{B}$ is the unique element in the $W$-orbit of $\nu$ that is dominant for $B$. Thus we have proved

THEOREM 6.3.1. - For any $x \in \widetilde{W}, \nu \in X_{*}(A)$ and $B \in \mathcal{B}(A)$ there is an equality

$$
\operatorname{dim} X_{x}\left(\varepsilon^{\nu}\right)=\sup _{w \in W}\left\{d\left(x, \varepsilon^{w \nu}, w B w^{-1}\right)-\left\langle\rho_{B}, \nu+\nu_{B}\right\rangle\right\} .
$$

$4^{\text {e }}$ SÉRIE - TOME $39-2006-\mathrm{N}^{\circ} 3$ 
In particular for $\nu=0$ we obtain

$$
\operatorname{dim} X_{x}(1)=\sup _{B \in \mathcal{B}(A)} d(x, 1, B) .
$$

This means in particular that $X_{x}\left(\varepsilon^{\nu}\right) \neq \emptyset$ if and only if there exists $w \in W$ such that $d\left(x, \varepsilon^{w \nu}, w B w^{-1}\right) \geqslant 0$.

\section{Computations}

\subsection{The algorithm}

In this section we present computational results on the non-emptiness and dimension of affine Deligne-Lusztig varieties in the affine flag manifold for a few groups of low rank. These results are computed according to Theorem 6.3.1 of Section 6.3, using the algorithm of Section 6.1 to compute the dimensions $d\left(x, \varepsilon^{w \nu}, w B w^{-1}\right)$.

Computations such as the ones presented here can in principle be done by hand, but their complexity leads us to use a computer program. The program is capable of representing all elements of the affine Weyl group of a root system up to a given length. It can also multiply these elements. The program can represent affine weights and coweights, as well as the action of the affine Weyl group on these weights and coweights.

Even using a computer, the computations presented here took some time. For instance, computing which alcoves correspond to non-empty affine Deligne-Lusztig varieties together with their dimensions in the $G_{2}$ case up to length 56 took several days on a medium-fast PC. To some extent, the program makes use of the automatical structure of the affine Weyl group (see [2], for instance). However, multiplication is carried out in the spirit of the method explained on p. 96 in [2], not by using a multiplier automaton (see Section 3 in [2]). Rewriting this part of the program would certainly lead to a significant speed-up. Memory consumption was never a problem.

\subsection{Reuman's conjecture}

We fix a Borel subgroup $B \subset G$ containing $A$, and let $\alpha_{i}$ denote the simple $B$-positive roots of $A$, i.e. the simple roots in $\operatorname{Lie}(B)$. Let $C_{0}$ denote the dominant Weyl chamber, which by definition is the set $\left\{x \in X_{*}(A)_{\mathbb{R}} \mid\left\langle\alpha_{i}, x\right\rangle>0, \forall i\right\}$. We call the unique alcove in the dominant Weyl chamber whose closure contains the origin the base alcove. As Iwahori subgroup $I$ we choose the Iwahori fixing this base alcove; $I$ is the inverse image of the opposite Borel group of $B$ under the projection $K \rightarrow G(\bar{k})$. We recall Reuman's conjecture from [32], starting with a definition.

Definition 7.2.1. - For $w \in W$ let $w\left(C_{0}\right)$ denote the Weyl chamber corresponding to $w$. For each simple positive root $\alpha_{i}$ of our root system, let $\varpi_{i}^{\vee}$ denote the corresponding fundamental coweight. Then we define the corresponding shrunken Weyl chamber $w\left(C_{0}\right)^{s h r} \subset w\left(C_{0}\right)$ by

$$
w\left(C_{0}\right)^{s h r}=w\left(C_{0}\right)+\sum_{\substack{i \\ w \alpha_{i}>0}} w \varpi_{i}^{\vee} .
$$

The union of the shrunken Weyl chambers is the set of all alcoves a in the standard apartment such that for each positive finite root $\alpha$, either the base alcove and a lie on opposite sides of the hyperplane $\{\alpha=0\}$, or they lie on opposite sides of the hyperplane $\{\alpha=1\}$. 
We denote by $\eta_{1}: W_{a} \rightarrow W$ the usual surjective homomorphism from the affine Weyl group to the finite Weyl group, and by $\eta_{2}: W_{a} \rightarrow W$ the map which associates to each alcove the finite Weyl chamber in which it lies.

Conjecture 7.2.2. - If $x$ lies in the shrunken Weyl chambers, then $X_{x}(1) \neq \emptyset$ if and only if

$$
\eta_{2}(x)^{-1} \eta_{1}(x) \eta_{2}(x) \in W \backslash \bigcup_{T \subsetneq S} W_{T},
$$

and in this case the dimension is given by

$$
\frac{1}{2}\left(\ell(x)+\ell\left(\eta_{2}(x)^{-1} \eta_{1}(x) \eta_{2}(x)\right)\right) .
$$

Here $S$ denotes the set of simple reflections, and for $T \subset S, W_{T}$ denotes the subgroup of $W$ generated by $T$. The set $W \backslash \bigcup_{T \subsetneq S} W_{T}$ is all elements of $W$ for which any reduced expression contains all simple reflections.

In [32] the conjecture was proved for the root systems $A_{2}$ and $C_{2}$. It is also true in all cases that were checked computationally: $G_{2}$ up to length $56, A_{3}$ up to length $38, C_{3}$ up to length 31 , $A_{4}$ up to length $28, C_{4}$ up to length 26.

For root systems of rank 2, results can be represented by a picture of the standard apartment (see the figures in [32] for $A_{2}, C_{2}$ and Fig. 1 for $G_{2}$ ). Alcoves corresponding to non-empty affine Deligne-Lusztig varieties are gray; those corresponding to empty affine Deligne-Lusztig varieties white; the base alcove is black. There is a dot at the origin. Non-empty affine DeligneLusztig varieties have their dimension on the corresponding alcove.

Outside the shrunken Weyl chambers, (7.2.1) is not equivalent to $X_{x}(1) \neq \emptyset$. For instance, all $X_{x}(1)$ with $x \in W$ are non-empty, even if $x \in \bigcup_{T \subsetneq S} W_{T}$. However, for $x \notin W$, (7.2.1) is a necessary criterion for non-emptiness in all the cases we checked. The version of this paper posted to the arXiv contains pictures illustrating the behavior in cases $A_{2}, C_{2}, G_{2}$. Except in the $A_{2}$ case, even if the affine Deligne-Lusztig variety for $x$ is non-empty, the predicted dimension may be wrong.

One can try to describe the set of alcoves for which the criterion (7.2.1) is not equivalent to the non-emptiness of the associated affine Deligne-Lusztig variety. For instance, in the $A_{2}$ case, for $x \notin W$, (7.2.1) wrongly predicts non-emptiness of $X_{x}(1)$ if and only if the alcove corresponding to $x$ lies outside the shrunken Weyl chambers and does not have the same number of vertices as the base alcove lying on the wall through the origin that it touches. We are not aware of a description of this kind that holds for more general root systems.

The criterion (7.2.1) is not invariant under the symmetry of root systems of type $A$ and $C$. It follows from [32] that for $A_{2}$, this realization leads to a complete description of which affine Deligne-Lusztig varieties are non-empty: $X_{x}(1) \neq \emptyset$ if and only if (7.2.1) holds for $x$ and its images under rotation by 120 and 240 degrees about the center of the base alcove. This does not work in the $C_{2}$ case, however.

\subsection{Lau's observation}

The following proposition is a direct consequence of [13, Prop. 5.4].

Proposition 7.3.1. - Let $G$ be semisimple and simply connected. Let $x \in W_{a}$, and let $\underline{x} \in N_{G(L)}(A)$ be a representative of $x$ which lies in $G(F)$. Then $x$ has finite order if and only if there exists an $y \in G(L)$ with $\underline{x}=y^{-1} \sigma(y)$. In particular, in this case $X_{x}(1)$ is non-empty.

$4^{\text {e }}$ SÉRIE - TOME $39-2006-\mathrm{N}^{\circ} 3$ 


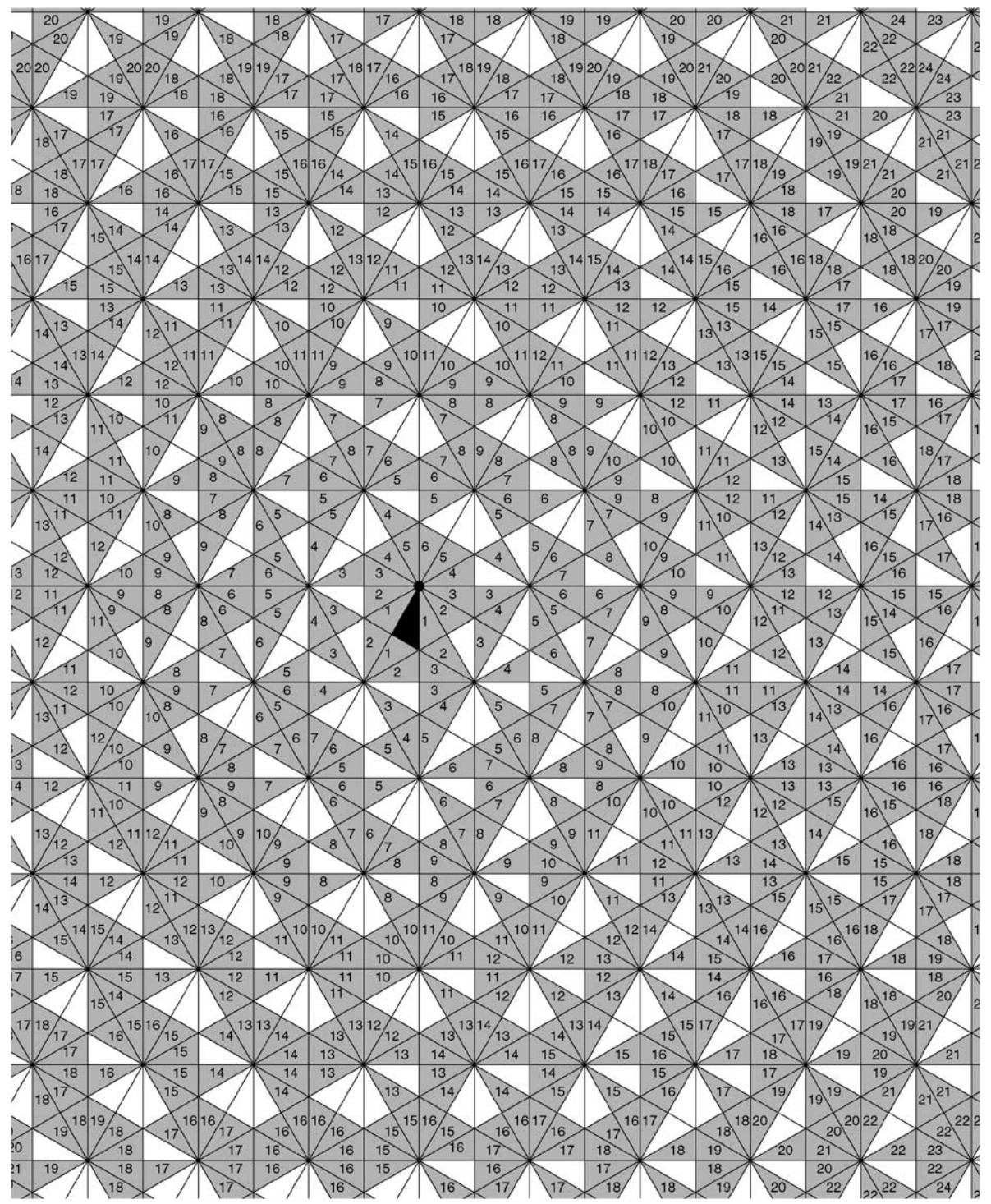

Fig. 1. Non-empty affine Deligne-Lusztig varieties and their dimensions, type $G_{2}, b=1$.

Starting from this proposition and analyzing Reuman's results in the $A_{2}$ case, Eike Lau observed that one can get another complete characterization of the non-empty affine DeligneLusztig varieties for $A_{2}$ :

Proposition 7.3.2. - Let $G=S L_{3}$, and let $x \in W_{a}, \ell(x)>1$. Then $X_{x}(1) \neq \emptyset$ if and only if there exists $n \geqslant 1$ with $\ell\left(x^{n}\right)<\ell(x)-1$.

One can prove this proposition by systematically analyzing Reuman's result for $S L_{3}$ (see [32]). It would be interesting to have a more conceptual proof. For other root systems, a similar criterion does not seem to hold (at least it is not sufficient to replace the offset -1 in the proposition by another integer). Nevertheless, this criterion still seems to give a reasonable approximation to the truth. It is possible that a minor modification of this criterion might hold more generally. 


\subsection{Partial folding sets}

Another approach to understanding the set of $x$ with non-empty affine Deligne-Lusztig variety is to consider the folding results separately for each direction. More precisely, for fixed $w \in W$, consider the set

$$
\left\{x \in \widetilde{W} ; d\left(x, 1, w B^{-} w^{-1}\right) \geqslant 0\right\},
$$

where $B^{-}$denotes the Borel group opposite to $B$. The version of this paper posted to the arXiv contains pictures illustrating these sets in the $A_{2}$ case. It is unclear whether these separate pieces are easier to understand than the entire result.

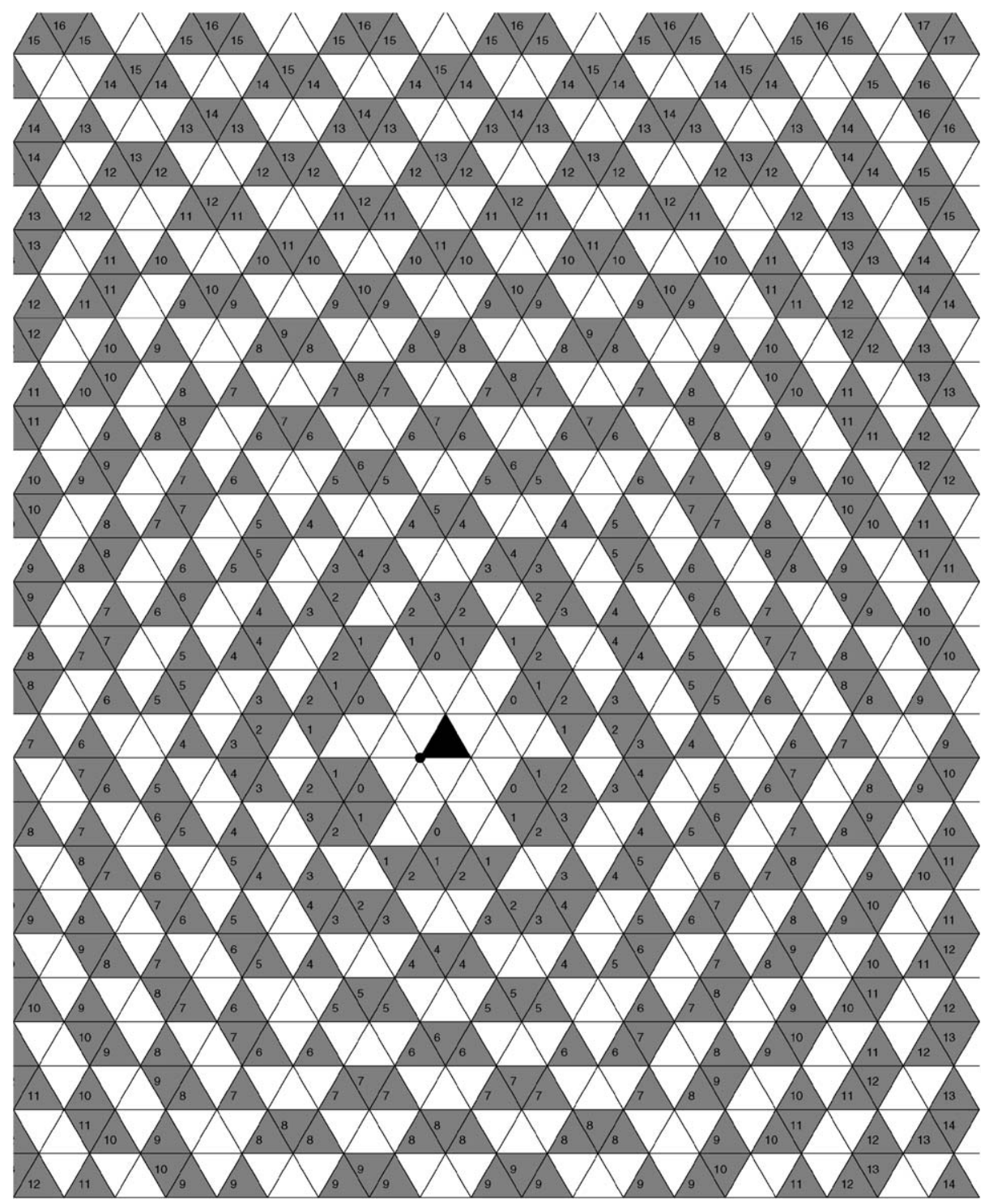

Fig. 2. Non-empty affine Deligne-Lusztig varieties and their dimensions, type $A_{2}, b=\varepsilon^{(1,0,-1)}$. 


\subsection{The case $b \neq 1$}

We give two examples with $b \neq 1$ : Fig. 2 shows results for $A_{2}, b=\varepsilon^{(1,0,-1)} ;$ Fig. 3 shows results for $C_{2}, b=\varepsilon^{(1,0)}$.

The following conjecture describes the dimension of $X_{x}\left(\varepsilon^{\nu}\right)$ for $x$ of sufficient length.

ConjeCture 7.5.1. - Let $G$ be one of the groups $S L_{2}, S L_{3}, S p_{4}$. Let $b=\varepsilon^{\nu}$, and write $\ell(b)$ for the length of the translation in $\widetilde{W}$ determined by $\nu$. Then there exists $n_{0}$ such that for all $x \in \widetilde{W}$ with $\ell(x) \geqslant n_{0}, X_{x}(b) \neq \emptyset$ if and only if $X_{x}(1) \neq \emptyset$. In this case,

$$
\operatorname{dim} X_{x}(b)=\operatorname{dim} X_{x}(1)-\frac{1}{2} \ell(b) .
$$

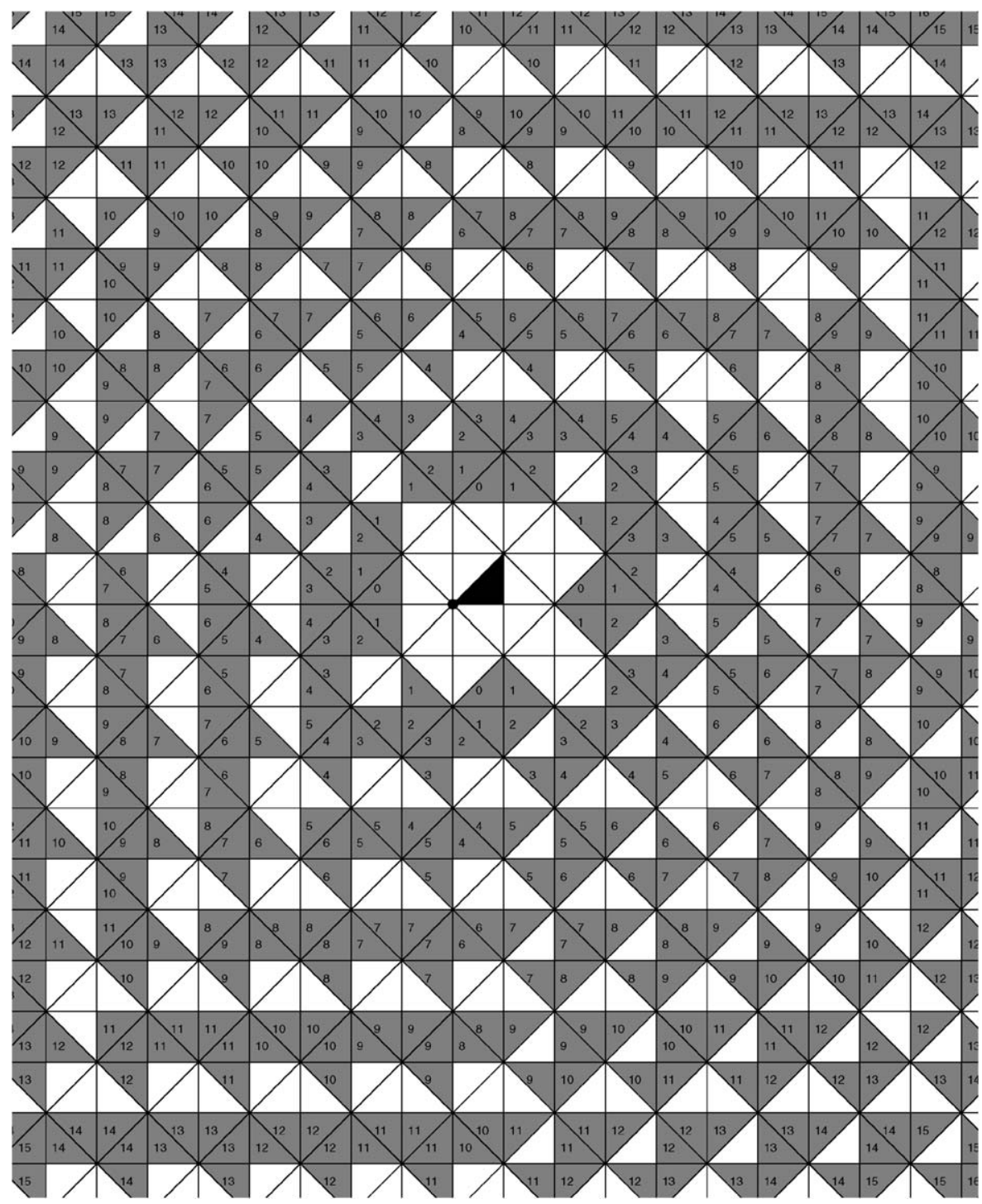

Fig. 3. Non-empty affine Deligne-Lusztig varieties and their dimensions, type $C_{2}, b=\varepsilon^{(1,0)}$. 
This conjecture describes $X_{x}(b)$ for $x$ in a larger region of the standard apartment than Reuman's Conjecture 7.1 in [32], but it is less precise because $n_{0}$ is not specified. The results in [31] support the new conjecture. It is possible that the conjecture holds for other groups, too. Various examples we have examined seem to indicate that there is a way to extend Reuman's conjecture to general $b$, but it is still premature to formulate a precise statement.

\section{Acknowledgements}

We thank the Rockefeller University Center for Physics and Biology for the use of their computer cluster.

\section{REFERENCES}

[1] Bruhat F., Tits J., Groupes réductifs sur un corps local. I, Inst. Hautes Études Sci. Publ. Math. 41 (1972) 5-251.

[2] Casselman W., Machine calculations in Weyl groups, Invent. Math. 116 (1994) 95-108.

[3] Chai C.-L., Newton polygons as lattice points, Amer. J. Math. 122 (5) (2000) 967-990.

[4] Dabrowski R., Comparison of the Bruhat and the Iwahori decompositions of a p-adic Chevalley group, J. Algebra 167 (3) (1994) 704-723.

[5] Deligne P., La conjecture de Weil II, Inst. Hautes Études Sci. Publ. Math. 52 (1980).

[6] Demazure M., Gabriel P., Groupes Algébriques: Tome I. Géométrie algébrique-GénéralitésGroupes commutatifs, Masson and CIE, Paris, 1970, 700 pp.+xxvi.

[7] DieudonnÉ J., Groupes de Lie et hyperalgèbres de Lie sur un corps de caractéristique $p>0$. VII, Math. Ann. 134 (1957) 114-133.

[8] Fargues L., Mantovan E., Variétés de Shimura, espaces de Rapoport-Zink et correspondances de Langlands locales, Astérisque 291 (2004).

[9] Haines T.J., On matrix coefficients of the Satake isomorphism: complements to the paper of Rapoport, Manuscripta Math. 101 (2000) 167-174.

[10] Haines T., KotTwitz R., PRASAd A., Iwahori-Hecke algebras, math.RT/0309168.

[11] KATO S., Spherical functions and a $q$-analogue of Kostant's weight multiplicity formula, Invent. Math. 66 (1982) 461-468.

[12] Kotтwitz R., Dimensions of Newton strata in the adjoint quotient of reductive groups, Pure Appl. Math. Q., in press, math.AG/0601196.

[13] Kotтwitz R., Isocrystals with additional structure, Compositio Math. 56 (1985) 201-220.

[14] Kotтwitz R., Isocrystals with additional structure. II, Compositio Math. 109 (1997) 255-339.

[15] Kоттwitz R., On the Hodge-Newton decomposition for split groups, Int. Math. Res. Not. 26 (2003) $1433-1447$.

[16] Kottwitz R., Rapoport M., On the existence of F-crystals, Comment. Math. Helv. 78 (2003) 153 184; math.NT/0202229.

[17] Leigh LuCARELli C., A converse to Mazur's inequality for split classical groups, J. Inst. Math. Jussieu 3 (2) (2004) 165-183; math.NT/0211327.

[18] LUSZTIG G., Singularities, character formulas, and a $q$-analog of weight multiplicities, in: Analyse et topologie sur les espaces singuliers, I-II (Luminy, 1981), Soc. Math. France, Paris, 1983, pp. 208229.

[19] Manin Y., Theory of commutative formal groups over fields of finite characteristic, Uspekhi Mat. Nauk 18 (6 (114)) (1963) 3-90 (in Russian); English translation in: Russian Math. Surveys 18 (6) (1963) 1-83.

[20] Mantovan E., On the cohomology of certain PEL type Shimura varieties, Duke Math. J. 129 (3) (2005) 573-610.

[21] Matsumoto H., Analyse harmonique dans les systèmes de Tits bornologiques de type affine, Springer Lecture Notes, vol. 590, Springer, Berlin, 1977.

[22] Mirkovic I., ViLONEN K., Geometric Langlands duality and representations of algebraic groups over commutative rings, math.RT/0401222v2.

$4^{\mathrm{e}}$ SÉRIE - TOME $39-2006-\mathrm{N}^{\circ} 3$ 
[23] Mirkovic I., Vilonen K., Perverse sheaves on affine Grassmannians and Langlands duality, Math. Res. Lett. 7 (1) (2000) 13-24.

[24] Mumford D., The Red Book of Varieties and Schemes, Lecture Notes in Math., vol. 1358, Springer, Berlin, 1988.

[25] NGô B.C., Polo P., Résolutions de Demazure affines et formule de Casselman-Shalika géométrique, J. Algebraic Geom. 10 (3) (2001) 515-547.

[26] OORT F., Foliations in moduli spaces of abelian varieties, J. Amer. Math. Soc. 17 (2) (2004) 267-296.

[27] RAPOPORT M., A positivity property of the Satake isomorphism, Manuscripta Math. 101 (2000) 153166.

[28] RAPOPORT M., A guide to the reduction modulo $p$ of Shimura varieties, Astérisque 298 (2005) 271318, math.AG/0205022.

[29] RAPOPORT M., RICHARTZ M., On the classification and specialization of F-isocrystals with additional structure, Compositio Math. 103 (1996) 153-181.

[30] Rapoport M., Zink T., Period Spaces for $p$-Divisible Groups, Ann. of Math. Stud., vol. 141, Princeton University Press, Princeton, NJ, 1996.

[31] Reuman D., Determining whether certain affine Deligne-Lusztig sets are non-empty, Thesis, Chicago, 2002, math.NT/0211434.

[32] REUMAN D., Formulas for the dimensions of some affine Deligne-Lusztig varieties, Michigan Math. J. 52 (2) (2004) 435-451.

[33] Viehmann E., Moduli spaces of $p$-divisible groups, math.AG/0502320.

[34] Viehmann E., The dimension of some affine Deligne-Lusztig varieties, Annales Sci. de l'E.N.S. 39 (3) (2006), math.AG/0510385.

(Manuscrit reçu le 9 mai 2005;

accepté, après révision, le 16 décembre 2005.)

\section{Ulrich GÖRTZ}

Mathematisches Institut,

Universität Bonn,

Beringstr. 1,

53115 Bonn, Germany

E-mail: ugoertz@math.uni-bonn.de

Thomas J. HAINES

Mathematics Department,

University of Maryland,

College Park, MD 20742-4015, USA

E-mail: tjh@math.umd.edu

Robert E. KотTwITZ

Department of Mathematics,

University of Chicago,

5734 University Avenue,

Chicago, IL 60637, USA

E-mail: kottwitz@math.uchicago.edu

Daniel C. REUMAN

Laboratory of Populations,

Rockefeller University, 1230 York Ave.,

New York, NY 10021, USA

E-mail: reumand@rockefeller.edu 